\title{
A THEORY OF CONGRUENCES AND BIRKHOFF'S THEOREM FOR MATROIDS
}

\author{
Stefan Veldsman $1,2, *$ \\ ${ }^{1}$ Nelson Mandela University, Port Elizabeth, SOUTH AFRICA \\ ${ }^{2}$ La Trobe University, Melbourne, AUSTRALIA \\ Communicated by Erhard Aichinger \\ Original Research Paper \\ Received: Jul 16, 2021 - Accepted: Sep 21, 2021 \\ First published online: October 28, 2021 \\ ๑) 2021 The Author(s)
}

\section{ABSTRACT}

A congruence is defined for a matroid. This leads to suitable versions of the algebraic isomorphism theorems for matroids. As an application of the congruence theory for matroids, a version of Birkhoff's Theorem for matroids is given which shows that every nontrivial matroid is a subdirect product of subdirectly irreducible matroids.

\section{KEYWORDS}

matroid; matroid congruence; matroid isomorphism theorems; subdirect product of matroids; subdirectly irreducible matroid

MATHEMATICS SUBJECT CLASSIFICATION (2020)

Primary 05B35; 05C25; 05C40; 08A30; Secondary

\section{INTRODUCTION}

A congruence is a fundamental notion and essential tool in algebra. Here we define and develop a theory of congruences for matroids. Although algebraically inspired, a matroid is not generally regarded as an algebraic structure and at an operational level has a strong graphical feel to it as well as a topological feel with the structure being determined by distinguished subsets. The recent development of a theory of congruences for graphs [2] and topological spaces [7], which closely resemble the algebraic theory of congruences, has prompted the question whether such a theory will be possible for matroids. As mentioned above, it is and it will be shown here that this theory for matroids leads to suitable versions of the algebraic notions associated with a congruence: kernels, quotients and the isomorphism theorems. Although the category of matroids do not have products, we will show that it is possible to define a subdirect product of matroids which leads to the notion of a subdirectly irreducible matroid. This enables a formulation and proof for matroids of the wellknown Birkhoff theorem from universal algebras: every nontrivial matroid is a subdirect product of subdirectly irreducible matroids. As a final application, two examples are given which suggest that it may be possible to define and develop a radical theory for matroids similar to that as for rings,

\footnotetext{
*Corresponding author. E-mail: veldsman@outlook.com
} 
groups, semigroups, graphs, topological spaces, etc. For graphs and topological spaces, this theory is known as a theory of connectednesses and disconnectednesses.

For matroids, we will mostly use the notions and notations as given by Oxley [6] and Gordon and McNulty [5]. But we do recall the basic concepts required. A matroid $M$ is a pair $M=(E, \mathcal{I})$ where $E$ is a finite nonempty set, called the ground set of $M, \mathcal{I}$ is a collection of subsets of $E$ called the independent subsets of $M$ and the following three conditions must be fulfilled:

(M1) $\varnothing \in \mathcal{I}$;

(M2) $\mathcal{I}$ is downwards closed, i.e. $A \subseteq B \in \mathcal{I}$ implies $A \in \mathcal{I}$;

(M3) (Independence augmentation axiom) For $A, B \in \mathcal{I}$ with $|A|<|B|$, there is a $b \in B$ - $A$ with $A \cup b \in \mathcal{I}$.

Here $|X|$ denotes the cardinality of the set $X$ and the set operations involving singleton sets like $\{b\}$ are usually written without the set brackets (as in (M3) above). When necessary to distinguish between different matroids, we will write $E_{M}$ and $\mathcal{I}_{M}$ for $E$ and $\mathcal{I}$ respectively. The following abuse of notation will often occur: For a matroid $M=(E, \mathcal{I})$, an element $a$ of $M$ or a subset $A$ of $M$ always refer to the ground set $E$ of $M$. Any subset $X$ of $E$ contains a maximal independent subset called a basis for $X$. The cardinality of this basis for $X$ is an invariant and is called the rank of $X$; written as $r(X)$. A basis in $M$ is a maximal independent subset of $M$ and $r(M)$ is the cardinality of any basis (and hence all) for $M$. If a subset of $E$ is not independent, it is called dependent and a minimal dependent subset of $M$ is called a circuit. A one element circuit is called a loop. The closure of $X$, written as $\bar{X}$, is defined by $\bar{X}=\{a \in E \mid r(X \cup a)=r(X)\}$ and $X$ is called a closed set (or flat) if $X=\bar{X}$. A submatroid $N$ of a matroid $M=(E, \mathcal{I})$ is a matroid $N=(F, \mathcal{J})$ where $F$ and $\mathcal{J}$ and nonempty subsets of $E$ and $\mathcal{I}$ respectively. Any nonempty subset $X$ of $E$ determines a submatroid $M_{X}=\left(X, \mathcal{I}_{X}\right)$ of $M=(E, \mathcal{I})$ where $\mathcal{I}_{X}:=\{I \subseteq X \mid I \in \mathcal{I}\}=\{I \cap X \mid I \in \mathcal{I}\}$. There are other submatroid structures on subsets of a matroid, but this will be our canonical submatroid of $M$ for any nonempty subset $X$ of $E$. It is sometimes called the restriction of $M$ to $X$ and denoted by $M \mid X$ (also called the deletion of $E-X$ from $M$ and denoted by $M-(E-X)$ ). The discrete matroid and indiscrete matroid on a nonempty set $E$ is given by $M=(E, \mathcal{I})$ where $\mathcal{I}$ is the collection of all the subsets of $E$ or $\mathcal{I}=\{\varnothing\}$ respectively. A trivial matroid is a matroid for which the ground set has exactly one element. There are two non-isomorphic trivial matroids on a ground set $\{a\}$ : the one with only the empty set as independent subset and the one with the empty set as well as $\{a\}$ as independent subsets (see the definition of isomorphic matroids below).

Mappings between matroids are important for what will be presented below and we recall relevant definitions and results in detail. For two matroids $M_{1}=\left(E_{1}, \mathcal{I}_{1}\right)$ and $M_{2}=\left(E_{2}, \mathcal{I}_{2}\right)$, a weak map $f: M_{1} \rightarrow M_{2}$ is a function $f: E_{1} \rightarrow E_{2}$ which satisfies the following condition: for any subset $A$ of $E_{1}$, if the restriction of $f$ to $A$, written as $\left.f\right|_{A}$, is injective and $f(A)$ is independent in $M_{2}$, then $A$ is independent in $M_{1}$. We will often use the following well-known characterizations of weak maps.

PROPOSITION 1.1. Let $M_{1}=\left(E_{1}, \mathcal{I}_{1}\right)$ and $M_{2}=\left(E_{2}, \mathcal{I}_{2}\right)$ be two matroids and $f: E_{1} \rightarrow E_{2}$ a function. The following conditions are equivalent:

(1) $f$ is a weak map.

(2) For every $A \subseteq M_{1}, r(f(A)) \leq r(A)$.

(3) For every dependent subset $D$ of $M_{1}$ with $\left.f\right|_{D}$ injective, $f(D)$ is dependent in $M_{2}$.

(4) For every circuit $C$ of $M_{1}$ with $\left.f\right|_{C}$ injective, $f(C)$ contains a circuit of $M_{2}$.

If $f: M_{1} \rightarrow M_{2}$ is a weak map and $\{x\}$ is a loop in $M_{1}$, then $\{f(x)\}$ is a loop in $M_{2}$. A strong map $f: M_{1} \rightarrow M_{2}$ is a function $f: E_{1} \rightarrow E_{2}$ for which $f^{-1}(A)$ is closed in $M_{1}$ for all closed subsets $A$ of $M_{2}$. A strong map is always also a weak map. It could well happen for a strong map that $f^{-1}(A)=\varnothing$ for a closed set $A$ of $M_{2}$, so a remark to clarify this case is in order. If $M_{1}$ has no loops, then $\bar{\varnothing}=\left\{x \in M_{1} \mid x\right.$ is a loop $\}=\varnothing$, so $\varnothing$ is closed in $M_{1}$. If $M_{1}$ has a loop, say $\{a\}$, then $\{f(a)\}$ is a loop in $M_{2}$. Since a closed set in a matroid contains all loops of the matroid, $f(a) \in A$ and in this case $f^{-1}(A) \neq \varnothing$. For strong maps we have:

PROPOSITION 1.2. Let $M_{1}=\left(E_{1}, \mathcal{I}_{1}\right)$ and $M_{2}=\left(E_{2}, \mathcal{I}_{2}\right)$ be two matroids and $f: E_{1} \rightarrow E_{2}$ a function. The following conditions are equivalent: 
(1) $f$ is a strong map.

(2) For every $A \subseteq M_{1}, f(\bar{A}) \subseteq \overline{f(A)}$.

(3) For $X \subseteq Y \subseteq M_{1}, r(f(Y))-r(f(X)) \leq r(Y)-r(X)$.

(4) For every independent set $A \subseteq M_{1}, f(\bar{A}) \subseteq \overline{f(A)}$.

The first two equivalences are well-known and the last is easy to verify. If $f: M_{1} \rightarrow M_{2}$ is a strong map, then $\overline{f^{-1}(B)} \subseteq f^{-1}(\bar{B})$ for any $B \subseteq M_{2}$ and for any $A \subseteq M_{1}, \overline{f(A)}=\overline{f(\bar{A})}, r(f(A))=r(f(\bar{A})$ ) and if $f(A)$ is closed, then $f(A)=f(\bar{A})$. An isomorphism $f: M_{1} \rightarrow M_{2}$ is a bijection $f: E_{1} \rightarrow E_{2}$ for which $I \subseteq M_{1}$ is independent if and only if $f(I)$ is independent in $M_{2}$. In such a case, $M_{1}$ is isomorphic to $M_{2}$ and it is denoted by $M_{1} \cong M_{2}$. For future reference, we record:

PROPOSITION 1.3. $f: M_{1} \rightarrow M_{2}$ is an isomorphism if and only if $f: E_{1} \rightarrow E_{2}$ is a bijection and any one of the six conditions listed below is fulfilled:

(1) Both $f$ and $f^{-1}$ are weak maps.

(2) For any $A \subseteq M_{1}, r(f(A))=r(A)$.

(3) For any $B \subseteq M_{2}, r(B)=r\left(f^{-1}(B)\right)$.

(4) For any $A \subseteq M_{1}, A$ is closed in $M_{1}$ if and only if $f(A)$ is closed in $M_{2}$.

(5) For any $B \subseteq M_{2}, B$ is closed in $M_{2}$ if and only if $f^{-1}(B)$ is closed in $M_{1}$.

(6) Both $f$ and $f^{-1}$ are strong maps.

The following expected results can easily be verified.

PROPOSITION 1.4. Let $M=(E, \mathcal{I})$ and $M^{\prime}=\left(E^{\prime}, \mathcal{I}^{\prime}\right)$ be matroids with $f: M \rightarrow M^{\prime}$ a weak map, $S$ a nonempty subset of $E$ with $M_{S}=\left(S, \mathcal{I}_{S}\right)$ the associated submatroid of $M$ and $\left(M^{\prime}\right)_{f(M)}=$ $\left(f(M),\left(\mathcal{I}^{\prime}\right)_{f(M)}\right)$ the submatroid of $M^{\prime}$ on $f(M)$. Then:

(1) The inclusion map $i: S \hookrightarrow E^{\prime}$ gives an injective weak map $i: M_{S} \rightarrow M^{\prime}$.

(2) The restriction $\left.f\right|_{S}: S \rightarrow E^{\prime}$ gives a weak map $\left.f\right|_{S}: M_{S} \rightarrow M^{\prime}$.

(3) $f: M \rightarrow\left(M^{\prime}\right)_{f(M)}$ is a surjective weak map.

We will have more to say about mappings in the next section.

\section{CONGRUENCES FOR A MATROID}

In this section, a congruence on a matroid will be defined and a basic theory of congruences for matroids, as it exists for universal algebra, will be developed. For this we need some preparations and we start with a well-known result. For completeness, we include one of the known proofs.

PROPOSITION 2.1. Let $M=(E, \mathcal{I})$ be a matroid with $f: E \rightarrow E^{\prime}$ a function, $E^{\prime} \neq \varnothing$. Then $M_{f}^{\prime}=$ $\left(E^{\prime}, f(\mathcal{I})\right)$ is a matroid where $f(\mathcal{I})=\{f(I) \mid I \in \mathcal{I}\}$.

Proof. Requirements (M1) and (M2) are straightforward to verify; we check (M3). Let $I, J \in \mathcal{I}$ with $|f(I)|<|f(J)|$. Without loss of generality we may choose $I$ and $J$ such that $|I|=|f(I)|,|J|=|f(J)|$ and $|I \cap J|$ as big as possible. Now $|I|<|J|$ and hence there is an element $j \in J-I$ with $I \cup j \in \mathcal{I}$. If $f(j) \in f(I)$, say $f(j)=f(i)$ for some $i \in I$, then $i \notin J$ since $f$ is injective on $J$. Let $I^{\prime}:=(I-i) \cup j$. Then $I^{\prime} \in \mathcal{I}$ and $f\left(I^{\prime}\right)=f(I)$ with $|I|=\left|I^{\prime}\right|=\left|f\left(I^{\prime}\right)\right|=|f(I)|$. But $\left|I^{\prime} \cap J\right|>|I \cap J|$ contradicts our choice of $I$ and $J$; hence $f(j) \notin f(I)$. Thus $f(j) \in f(J)-f(I)$ and $f(I) \cup f(j)=f(I \cup j)$ with $I \cup j \in \mathcal{I}$ and (M3) follows.

The function $f$ as in the above statement need not give a weak map $f: M \rightarrow M_{f}^{\prime}$. Following the next proposition, we will see when it does.

PROPOSITION 2.2. Let $M=(E, \mathcal{I})$ and $M^{\prime}=\left(E^{\prime}, \mathcal{I}^{\prime}\right)$ be matroids and with $f: E \rightarrow E^{\prime}$ a function. Let $\mathbb{I}_{f}:=\left\{I \subseteq M|f|_{I}\right.$ is injective and $\left.f(I) \in \mathcal{I}^{\prime}\right\}$. Then:

(1) $M_{f}:=\left(E, \mathbb{I}_{f}\right)$ is a matroid, $f: M_{f} \rightarrow M^{\prime}$ is a weak map and $f\left(\mathbb{I}_{f}\right) \subseteq \mathcal{I}^{\prime}$.

(2) If $f$ is surjective, then $f\left(\mathbb{I}_{f}\right)=\mathcal{I}^{\prime}$.

(3) $f: M \rightarrow M^{\prime}$ is a weak map if and only if $\mathbb{I}_{f} \subseteq \mathcal{I}$.

(4) If $f$ is a surjective weak map, then $\mathcal{I}^{\prime} \subseteq f(\mathcal{I})$. 
Proof. (1) To show that $M_{f}$ is a matroid, we only verify (M3). Let $A, B \in \mathbb{I}_{f}$ with $|A|<|B|$. Then both $\left.f\right|_{A}$ and $\left.f\right|_{B}$ are injective and $f(A), f(B) \in \mathcal{I}^{\prime}$. Since $|f(A)|=|A|<|B|=|f(B)|$, there is a $y \in f(B)-f(A)$ with $f(A) \cup y \in \mathcal{I}^{\prime}$. Then $y=f(b)$ for some $b \in B-A$ and $f(A \cup b)=f(A) \cup y \in \mathcal{I}^{\prime}$. Since $\left.f\right|_{A \cup b}$ is injective, $A \cup b \in \mathbb{I}_{f}$ and hence $M_{f}$ a matroid follows. The definition of $\mathbb{I}_{f}$ ensures that $f: M_{f} \rightarrow M^{\prime}$ is a weak map and $f\left(\mathbb{I}_{f}\right) \subseteq \mathcal{I}^{\prime}$.

(2) Suppose $f$ is surjective and let $I \in \mathcal{I}^{\prime}$. By the surjectivity of $f$, we can choose $J \subseteq E$ such that $\left.f\right|_{J}: J \rightarrow f(J)=I$ is injective. Then $J \in \mathbb{I}_{f}$ and $I \in f\left(\mathbb{I}_{f}\right)$. By (1), $f\left(\mathbb{I}_{f}\right)=\mathcal{I}^{\prime}$.

(3) Assume $f: M \rightarrow M^{\prime}$ is a weak map. Let $I \in \mathbb{I}_{f}$. Then $I \in \mathcal{I}$ by the definition of a weak map. Conversely, suppose $\mathbb{I}_{f} \subseteq \mathcal{I}$. Choose $I \subseteq M$ such that $\left.f\right|_{I}: I \rightarrow f(I)$ is injective with $f(I) \in \mathcal{I}^{\prime}$. Then $I \in \mathbb{I}_{f} \subseteq \mathcal{I}$ and $f$ a weak map follows.

(4) If $f: M \rightarrow M^{\prime}$ is a surjective weak map, then (2) and (3) give $\mathcal{I}^{\prime}=f\left(\mathbb{I}_{f}\right) \subseteq f(\mathcal{I})$.

A note of caution: the definition of $\mathbb{I}_{f}$ depends on the map $f$ as well as the matroid structure of the codomain. Reference to this in the notation $\mathbb{I}_{f}$ has been omitted for notational simplicity since in most cases there is no reason for confusion. For the map $f: M \rightarrow M^{\prime}$ as specified in Proposition 2.1, the requirement to be a weak map is given below. But we first fix some terminology. For a set $E$, a subset $A$ of $E$ and an equivalence relation $\sim$ on $E$, by a transversal for $A$ with respect to $\sim$ we understand a subset $T_{A}$ of $E$ which contains exactly one representative from every equivalence class which meets $A$. Note that we do not require a transversal $T_{A}$ for $A$ to be a subset of $A$. Below we will use the equivalence relation $\sim_{f}$ determined by a function $f$ with domain $E: a \sim_{f} b \Leftrightarrow f(a)=f(b)$. This is an equivalence on $E$ with equivalence classes $[a]_{f}=f^{-1}(f(a))$ for all $a \in E$. Note that for $I \subseteq E$, $\left.f\right|_{I}$ is injective if and only if for all $a \in I,[a]_{f} \cap I=\{a\}$. In the formulation of the next proposition, we will use the result from Proposition 2.1.

PROPOSITION 2.3. Let $M=(E, \mathcal{I})$ be a matroid, $E^{\prime}$ a nonempty set and $f: E \rightarrow E^{\prime}$ a function. Let $M_{f}^{\prime}$ be the matroid $M_{f}^{\prime}=\left(E^{\prime}, f(\mathcal{I})\right)$ and let $\mathbb{I}_{f}:=\left\{I \subseteq M|f|_{I}\right.$ is injective and $\left.f(I) \in f(\mathcal{I})\right\}$. Then:

(1) $\mathbb{I}_{f}=\left\{I \subseteq M \mid I\right.$ is a transversal for some $J \in \mathcal{I}$ with respect to $\left.\sim_{f}\right\}$ and $f\left(\mathbb{I}_{f}\right)=f(\mathcal{I})$.

(2) $f: M \rightarrow M_{f}^{\prime}$ is a weak map if and only if $\mathbb{I}_{f} \subseteq \mathcal{I}$.

(3) $\mathcal{I} \subseteq \mathbb{I}_{f}$ if and only if for every $a \in I \in \mathcal{I},[a] \cap I=\{a\}$.

Proof. (1) Both these statements follow directly from the relevant definitions and (2) holds by Proposition 2.2(3). For (3), see the lines preceding this proposition.

The next lemma is required for one last result before we start with the congruence theory.

LEMMA 2.4. Let $M=(E, \mathcal{I})$ and $M^{\prime}=\left(E^{\prime}, \mathcal{I}^{\prime}\right)$ be matroids with $f: M \rightarrow M^{\prime}$ a weak map. Let $B \subseteq U \subseteq E$ with $\left.f\right|_{B}$ injective. Then $B$ is a basis for $U$ in the matroid $M_{f}=\left(E, \mathbb{I}_{f}\right)$ if and only if $f(B)$ is a basis for $f(U)$ in the matroid $M^{\prime}=\left(E^{\prime}, \mathcal{I}^{\prime}\right)$.

Proof. Suppose $B$ is a basis for $U$ in the matroid $M_{f}=\left(E, \mathbb{I}_{f}\right)$. Then $B \in \mathbb{I}_{f}$ which means $\left.f\right|_{B}$ is injective and $f(B) \in \mathcal{I}^{\prime}$. If $f(B)$ is not a basis for $f(U)$, there is an $u \in U$ such that $f(u) \notin f(B)$ and $f(B) \cup f(u) \in \mathcal{I}^{\prime}$. Hence $u \notin B$ and $\left.f\right|_{B \cup u}$ is injective with $f(B \cup u) \in \mathcal{I}^{\prime}$. This gives $B \cup u \in \mathbb{I}_{f}$ which contradicts $B$ is a basis for $U$ in the matroid $M_{f}$. Thus $f(B)$ is a basis for $f(U)$. Conversely, suppose $f(B)$ is a basis for $f(U)$ in $M^{\prime}=\left(E^{\prime}, \mathcal{I}^{\prime}\right)$. Then $f(B) \in \mathcal{I}^{\prime}$ and by assumption, $\left.f\right|_{B}$ is injective. Thus $B \in \mathbb{I}_{f}$. If $B$ is not a basis for $U$ in $M_{f}=\left(E, \mathbb{I}_{f}\right)$, there is an $u \in U-B$ with $B \cup u \in \mathbb{I}_{f}$. By definition of $\mathbb{I}_{f},\left.f\right|_{B \cup u}$ is injective and $f(B \cup u) \in \mathcal{I}^{\prime}$. Since $f(u) \notin f(B)$, this contradicts $f(B)$ a basis for $f(U)$.

PROPOSITION 2.5. Let $M=(E, \mathcal{I})$ and $M^{\prime}=\left(E^{\prime}, \mathcal{I}^{\prime}\right)$ be matroids with $f: M \rightarrow M^{\prime}$ a weak map. Then $f$ is a strong map if and only if the following condition is satisfied: whenever $U \subseteq M$ is independent, $x \in M$ with $f(x) \notin f(U)$ and $B \subseteq U$ a basis for $U$ in the matroid $M_{f}=\left(E, \mathbb{I}_{f}\right)$, then $B \cup x \in \mathbb{I}_{f}$ implies $U \cup x \in \mathcal{I}$.

Proof. Suppose $f$ is a strong map. Let $U \subseteq M$ be independent and let $x \in M$ with $f(x) \notin f(U)$. Let $B$ be a basis for $U$ in $M_{f}=\left(E, \mathbb{I}_{f}\right)$ and suppose $B \cup x \in \mathbb{I}_{f}$. This means $\left.f\right|_{B \cup x}$ is injective and $f(B \cup x) \in \mathcal{I}^{\prime}$. By Lemma 2.4, we know $f(B)$ is a basis for $f(U)$. Seeking a contradiction, let us suppose 
that $U \cup x \notin \mathcal{I}$. Then $r(U \cup x)=r(U)$ since $U$ is independent. This means $x \in \bar{U}$ and since $f$ is a strong map, $f(x) \in \overline{f(U)}=\overline{f(B)}$ (see Proposition 1.2(2)); the last equality holds since $f(B)$ is a basis for $f(U)$. Thus $f(x) \in \overline{f(B)}-f(B)$ and so $|B \cup x|=|f(B \cup x)|=r(f(B \cup x))=r(f(B))=|f(B)|=|B|$. This is only possible if $x \in B$ which gives the contradiction $f(x) \in f(B) \subseteq f(U)$. We can conclude that $U \cup x \in \mathcal{I}$.

Conversely, suppose the condition holds. Let $U \subseteq M$ be independent. To conclude that $f$ is a strong map, we show $f(\bar{U}) \subseteq \overline{f(U)}$. Let $x \in \bar{U}$. Since $f(U) \subseteq \overline{f(U)}$, we are done if $f(x) \in f(U)$; so suppose $f(x) \notin f(U)$. Hence $x \notin U$ and in particular, this means $x \notin B$ and $f(x) \notin f(B)$ where $B$ is a basis for $U$ in the matroid $M_{f}=\left(E, \mathbb{I}_{f}\right)$. By Lemma 2.4, $f(B)$ is a basis for $f(U)$ and hence $\overline{f(U)}=\overline{f(B)}$. If $f(x) \notin \overline{f(U)}=\overline{f(B)}$, then $r(f(B \cup x))=r(f(B) \cup f(x))>r f(B))=|f(B)|$. Thus $f(B \cup x) \in \mathcal{I}^{\prime}$. Now $B \in \mathbb{I}_{f}$ means $\left.f\right|_{B}$ is injective and since $x \notin B$ and $f(x) \notin f(B)$, we have $\left.f\right|_{B \cup x}$ is injective. Consequently $B \cup x \in \mathbb{I}_{f}$ and by the assumption, $U \cup x \in \mathcal{I}$. But then $r(U \cup x)=|U \cup x|>|U|=r(U)=r(U \cup x)$ since $x \in \bar{U}$. This contradiction enables us to conclude that $f(x) \in \overline{f(U)}$ and that $f$ is a strong map.

From universal algebra we know that a congruence on an algebra is an equivalence relation on the algebra which is well-behaved with respect to the algebraic operations. Thus, for a matroid we want an equivalence relation on the ground set which, in some sense, is well-behaved with respect to the independent subsets. For an equivalence $\sim$ on a set $E$, the equivalence class of an element $a \in E$ will be denoted by $[a]$. When necessary, $[a]$ will be written with a subscript to clarify matters when there are more than one equivalence under discussion. The function $\pi: E \rightarrow\{[a] \mid a \in E\}$ is the obvious surjective quotient map $\pi(a)=[a]$ for all $a \in E$.

DEFINITION 2.6. A congruence $\gamma$ on a matroid $M=(E, \mathcal{I})$ is a pair $\gamma=(\sim, \mathbb{I})$, where

(C1) is an equivalence relation on $E$;

(C2) I is a subset of $\mathcal{I}$, called the congruence independent set, and $M_{\gamma}:=(E, \mathbb{I})$ is a matroid;

(C3) (Substitution Property) If $I=\left\{a_{1}, a_{2}, \ldots, a_{n}\right\} \in \mathbb{I}$ and $J=\left\{b_{1}, b_{2}, \ldots, b_{n}\right\} \subseteq E$ with $|I|=|J|=n$ and $a_{i} \sim b_{i}$ for all $i=1,2, \ldots, n$, then $J \in \mathbb{I}$;

(C4) For all $a \in I \in \mathbb{I},[a] \cap I=\{a\}$.

A strong congruence is a congruence which fulfills the additional condition:

(C5) If $U \subseteq M$ is independent, $x \in M$ with $[x] \cap U=\varnothing$ and $B \subseteq U$ a basis for $U$ in the matroid $M_{\gamma}=(E, \mathbb{I})$, then $B \cup x \in \mathbb{I}$ implies $U \cup x \in \mathcal{I}$.

For a congruence on a matroid, the associated equivalence classes will sometimes be called the congruence classes. As examples, we now only give the two trivial congruences on a matroid; later more will be seen. In any case, the next proposition will give the prototype of all matroid congruences. The identity congruence $\iota_{M}=(\approx, \mathbb{I})$ on $M=(E, \mathcal{I})$ has $a \approx b$ if and only if $a=b$ for $a, b \in E$ and the congruence independent set II is just the set of all independent sets $\mathcal{I}$ of $M$. The universal congruence $v_{M}=(\rightsquigarrow, \mathbb{I})$ on $M$ has $a \rightsquigarrow b$ for all $a, b \in E$ and $\mathbb{I}=\{\varnothing\}$. Both these congruences are strong congruences.

PROPOSITION 2.7. Let $M=(E, \mathcal{I})$ and $M^{\prime}=\left(E^{\prime}, \mathcal{I}^{\prime}\right)$ be matroids with $f: M \rightarrow M^{\prime}$ a weak map. Then $\operatorname{ker} f:=\left(\sim_{f}, \mathbb{I}_{f}\right)$ with $a \sim_{f} b \Leftrightarrow f(a)=f(b), a, b \in E$ and $\mathbb{I}_{f}:=\left\{I \subseteq M|f|_{I}\right.$ is injective and $\left.f(I) \in \mathcal{I}^{\prime}\right\}$ is a congruence on $M$. If $f$ is a strong map, then $\operatorname{ker} f$ is a strong congruence.

Proof. (C1) is straightforward and (C2) follows from Proposition 2.2(3) and (1). For (C3), we use Proposition 2.3(1). Let $I=\left\{a_{1}, a_{2}, \ldots, a_{n}\right\} \in \mathbb{I}_{f}$ and $J=\left\{b_{1}, b_{2}, \ldots, b_{n}\right\} \subseteq E$ with $|I|=|J|=n$ and $a_{i} \sim_{f} b_{i}$ for all $i=1,2, \ldots, n$. Then $J$ is a transversal for $I$ with respect to $\sim_{f}$ and hence in $\mathbb{I}_{f}$. Again, using Proposition 2.3(1), we get (C4). If $f$ is a strong map, then (C5) follows from Proposition 2.5. Indeed, for any $U \subseteq M$ and $x \in M,[x]_{f}=f^{-1}(f(x))$ and so $[x]_{f} \cap U=\varnothing$ if and only if $f(x) \notin f(U)$.

It can easily be verified that a weak map $f: M \rightarrow M^{\prime}$ is injective if and only if $\operatorname{ker} f=\left(\approx, \mathbb{I}_{f}\right)$ with $\mathbb{I}_{f}=\left\{f^{-1}(J) \mid J \in \mathcal{I}^{\prime}\right\}$. On occasion it is necessary to compare congruences. An ordering $ᄃ$ on congruences is defined as follows. Let $\alpha=\left(\sim \alpha, \mathbb{I}_{\alpha}\right)$ and $\beta=\left(\sim \beta, \mathbb{I}_{\beta}\right)$ be two congruences on a matroid $M=(E, \mathcal{I})$. Then $\alpha$ is contained in $\beta$, written as $\alpha \subseteq \beta$, if $\sim \alpha \subseteq \sim \beta$ and $\mathbb{I}_{\beta} \subseteq \mathbb{I}_{\alpha}$. Equality $\alpha=\beta$, 
of course, means $\sim \alpha=\sim \beta$ and $\mathbb{I}_{\alpha}=\mathbb{I}_{\beta}$; thus $\alpha=\beta \Leftrightarrow \alpha \sqsubseteq \beta$ and $\beta \sqsubseteq \alpha$. For any congruence $\gamma$ on a matroid $M, \iota_{M} \sqsubseteq \gamma \sqsubseteq v_{M}$. This definition of containment is in part motivated by the next result.

PROPOSITION 2.8. Let $M=(E, \mathcal{I}), M_{1}=\left(E_{1}, \mathcal{I}_{1}\right)$ and $M_{2}=\left(E_{2}, \mathcal{I}_{2}\right)$ be three matroids. Let $f: M \rightarrow M_{1}$ and $g: M \rightarrow M_{2}$ be two surjective weak maps. Then ker $f \sqsubseteq$ ker $g$ if and only if there is a unique weak map $h: M_{1} \rightarrow M_{2}$, necessarily surjective, such that $h \circ f=g$.

Proof. Let ker $f=\left(\sim f, \mathbb{I}_{f}\right)$ and ker $g=\left(\sim g, \mathbb{I}_{g}\right)$. Suppose a weak map $h$ with $h \circ f=g$ exists. If $a, b \in M$ with $a \sim_{f} b$, then $f(a)=f(b)$ and so $g(a)=g(b)$; hence $a \sim_{g} b$. Let $I \in \mathbb{I}_{g}$. Then $\left.g\right|_{I}: I \rightarrow g(I)=h(f(I)) \in \mathcal{I}_{2}$ is injective. This means $\left.h\right|_{f(I)}: f(I) \rightarrow h(f(I)) \in \mathcal{I}_{2}$ is injective and so $f(I) \in \mathcal{I}_{1}$. But then, since $\left.f\right|_{I}: I \rightarrow f(I)$ is injective, $I \in \mathbb{I}_{f}$ and ker $f \sqsubseteq$ ker $g$ follows. Conversely, suppose ker $f \sqsubseteq \operatorname{ker} g$. Define a function $h: E_{1} \rightarrow E_{2}$ as follows: Let $y \in E_{1}$ and then choose $x \in E$ with $f(x)=y$. Let $h(y)=g(x)$. The mapping $h$ is well-defined: Indeed, if $f(x)=y=f\left(x^{\prime}\right)$, then $x \sim f x^{\prime}$ and from $\sim f \subseteq \sim g$ we get $g(x)=g\left(x^{\prime}\right)$. The surjectivity of $h$ and the equality $h \circ f=g$ are clear, so we check that $h$ is a weak map. Let $J \subseteq E_{1}$ such that $\left.h\right|_{J}: J \rightarrow h(J) \in \mathcal{I}_{2}$ is injective. The surjectivity of $f$ gives $K \subseteq E$ such that $\left.f\right|_{K}: K \rightarrow f(K)=J$ is injective. Then $\left.g\right|_{K}=\left.(h \circ f)\right|_{K}$ is injective and $h(f(K))=h(J) \in \mathcal{I}_{2}$. Thus $K \in \mathbb{I}_{g} \subseteq \mathbb{I}_{f}$. This means $\left.f\right|_{K}: K \rightarrow f(K)=J \in \mathcal{I}_{1}$. Thus $h$ is a weak map.

The next result is really the essence of the concept of a congruence. We have already seen that any weak map gives rise to a congruence namely its kernel (Proposition 2.7). Below we will see that any congruence is the kernel of a weak map, namely the kernel of the quotient map determined by the congruence.

PROPOSITION 2.9. Let $M=(E, \mathcal{I})$ be a matroid and let $\gamma=\left(\sim \gamma, \mathbb{I}_{\gamma}\right)$ be a congruence on $M$. Let $M / \gamma:=(E / \gamma, \mathcal{I} / \gamma)$ where $E / \gamma:=\{[a] \mid a \in M\}$ and $\mathcal{I} / \gamma:=\left\{\pi(I) \mid I \in \mathbb{I}_{\gamma}\right\}$. Then $M / \gamma$ is a matroid and $\pi: M \rightarrow M / \gamma$ is a surjective weak map with $\operatorname{ker} \pi=\gamma$. If $\gamma$ is a strong congruence, then $\pi$ is a strong map.

Proof. Clearly $E / \gamma$ is a nonempty set. By definition of a congruence, $\left(E, \mathbb{I}_{\gamma}\right)$ is a matroid and $M / \gamma=$ $(E / \gamma, \mathcal{I} / \gamma)$ a matroid follows from Proposition 2.1. Next it is shown that $\pi: M \rightarrow M / \gamma$ is a weak map. Choose $J:=\left\{b_{1}, b_{2}, \ldots, b_{n}\right\} \subseteq E$ such that $\left.\pi\right|_{J}: J \rightarrow \pi(J) \in \mathcal{I} / \gamma$ is injective. By definition, $\pi(J)=\pi(I)$ for some $I:=\left\{a_{1}, a_{2}, \ldots, a_{m}\right\} \in \mathbb{I}_{\gamma}$. Condition (C4) ensures $\left.\pi\right|_{I}: I \rightarrow \pi(I)$ is injective. Thus $n=m$ and from $\pi(J)=\pi(I)$ we get, with some reordering if necessary, $a_{i} \sim_{\gamma} b_{i}$ for all $i=1,2, \ldots, n$. Since $I \in \mathbb{I}_{\gamma}$, the Substitution Property gives $J \in \mathbb{I}_{\gamma} \subseteq \mathcal{I}$. We conclude that $\pi: M \rightarrow M / \gamma$ is a weak map. Let ker $\pi=\left(\sim_{\pi}, \mathbb{I}_{\pi}\right)$. We show $\gamma=\operatorname{ker} \pi$. For $a, b \in M, a \sim_{\gamma} b \Leftrightarrow \pi(a)=[a]_{\gamma}=[b]_{\gamma}=\pi(b) \Leftrightarrow a \sim_{\pi} b$. By definition, $\mathbb{I}_{\pi}=\left\{J \subseteq M|\pi|_{J}: J \rightarrow \pi(J)\right.$ is injective and $\left.\pi(J) \in \mathcal{I} / \gamma\right\}$. This means, for $J \in \mathbb{I}_{\pi}$, $\pi(J)=\pi(I)$ for some $I \in \mathbb{I}_{\gamma}$. Using the Substitution Property and an argument similar to the above, we get $J \in \mathbb{I}_{\gamma}$. Conversely, if $J \in \mathbb{I}_{\gamma}$, then by (C4) we have $\left.\pi\right|_{J}: J \rightarrow \pi(J)$ is injective and by definition, $\pi(J) \in \mathcal{I} / \gamma$. This means $J \in \mathbb{I}_{\pi}$. Thus $\mathbb{I}_{\gamma}=\mathbb{I}_{\pi}$ and so ker $\pi=\gamma$. If $\gamma$ is a strong congruence, then $\pi: M \rightarrow M / \gamma$ a strong map follows from Proposition 2.5.

We now formulate and proof for matroids what is known as the isomorphism theorems for universal algebra (groups, rings, etc.).

THEOREM 2.10 (First Isomorphism Theorem). Let $M=(E, \mathcal{I})$ and $M^{\prime}=\left(E^{\prime}, \mathcal{I}^{\prime}\right)$ be matroids with $f: M \rightarrow$ $M^{\prime}$ a surjective weak map with $\gamma=\operatorname{ker} f$. Then $M / \gamma \cong M^{\prime}$.

Proof. For the two surjective weak maps $f: M \rightarrow M^{\prime}$ and $\pi: M \rightarrow M / \gamma, \operatorname{ker} \pi=\gamma=\operatorname{ker} f$ and by Proposition 2.8 there are two surjective weak maps $h: M^{\prime} \rightarrow M / \gamma$ and $h^{\prime}: M / \gamma \rightarrow M^{\prime}$ with $h \circ f=\pi$ and $h^{\prime} \circ \pi=f$. Then $h \circ h^{\prime}=1_{M / \gamma}$ and $h^{\prime} \circ h=1_{M^{\prime}}$ which give $M / \gamma \cong M^{\prime}$ by Proposition 1.3.

The two obvious quotient matroids are described next. Let $M=(E, \mathcal{I})$ be a matroid. Then $M / \iota_{M} \cong M$; in fact, for a congruence $\gamma$ on $M, M / \gamma \cong M \Longleftrightarrow \gamma=\iota_{M}$. There are only two congruences possible with the universal equivalence $\leadsto$ as equivalence relation on a matroid $M=(E, \mathcal{I})$ namely $\mathbb{I}=\{\varnothing\}$ or $\mathbb{I}=\{\varnothing\} \cup\{\{b\} \mid b \in E\}$. Indeed, if $\gamma=(\leftrightarrow \rightsquigarrow, \mathbb{I})$ is a congruence on $M$ with $\mathbb{I} \neq\{\varnothing\}$, choose 
$\varnothing \neq I \in \mathbb{I}$, say $a \in I$. By (C4), $\{a\}=[a] \cap I=E \cap I=I$, and by (C3), it then follows that $\{b\} \in \mathbb{I}$ for all $b \in M$. Thus, for a congruence $\gamma$ on the matroid $M, M / \gamma$ is trivial if and only if $\gamma$ is one of the two congruences $\gamma=(\leftrightarrow, \mathbb{I})$ for the two possibilities of I described above. If $M$ has a loop, then $\gamma=v_{M}$, so $\mathbb{I}=\{\varnothing\} \cup\{\{b\} \mid b \in E\}$ can only occur when $M$ has no loops.

For the Second Isomorphism Theorem we need to set the scene. Let $M=(E, \mathcal{I})$ be a matroid, let $\gamma=\left(\sim \gamma, \mathbb{I}_{\gamma}\right)$ be a congruence on $M$ and let $M_{S}:=\left(S, \mathcal{I}_{S}\right)$ be a submatroid of $M$ (i.e., the restriction of $M$ to $S)$. We can restrict the congruence $\gamma$ on $M$ to a congruence $\gamma_{S}:=\left(\sim_{\gamma_{S}}, \mathbb{I}_{\gamma_{S}}\right)$ on the matroid $M_{S}$ where, for $a, b \in S, a \sim_{\gamma_{S}} b \Leftrightarrow a \sim_{\gamma} b$ and $\mathbb{I}_{\gamma_{S}}:=\left\{I \cap S \mid I \in \mathbb{I}_{\gamma}\right\}$. Sometimes $\gamma_{S}$ is written as $\gamma_{S}=S \cap \gamma$. That this is actually a congruence, is given in the next result.

PROPOSITION 2.11. Let $M=(E, \mathcal{I})$ be a matroid and let $\gamma=\left(\sim_{\gamma}, \mathbb{I}_{\gamma}\right)$ be a congruence on $M$. Let $M_{S}=\left(S, \mathcal{I}_{S}\right)$ be a submatroid of $M$. Then $\gamma_{S}=\left(\sim_{\gamma_{S}}, \mathbb{I}_{\gamma_{S}}\right)$ is a congruence on the matroid $M_{S}$. If $\gamma$ is a strong congruence, then so is $\gamma_{S}$.

Proof. Since $M \gamma=\left(E, \mathbb{I}_{\gamma}\right)$ is a matroid, we know that $\left(S, \mathbb{I}_{\gamma_{S}}\right)$ is a matroid and $\mathbb{I}_{\gamma_{S}} \subseteq \mathcal{I}_{S}$. Clearly $\sim \gamma_{S}$ is an equivalence on $S$. These opening statements give (C1) and (C2) for showing $\gamma_{S}$ is a matroid on $M_{S}$. For (C3), let $I=\left\{a_{1}, a_{2}, \ldots, a_{n}\right\} \in \mathbb{I}_{\gamma_{S}}, J=\left\{b_{1}, b_{2}, \ldots, b_{n}\right\} \subseteq S$ with $|I|=|J|=n$ and $a_{i} \sim \gamma_{S} b_{i}$ for all $i=1,2, \ldots, n$. Since $\mathbb{I}_{\gamma_{S}} \subseteq \mathbb{I}_{\gamma}$ and $\gamma$ is a congruence, $J \in \mathbb{I}_{\gamma}$ holds and hence $J=J \cap S \in \mathbb{I}_{\gamma_{S}}$. For (C4), let $a \in I=J \cap S, J \in \mathbb{I}_{\gamma}$. Then $[a]_{\gamma} \cap J=\{a\}$ which gives $[a]_{\gamma_{S}} \cap I=\left([a]_{\gamma} \cap S\right) \cap I \subseteq[a]_{\gamma} \cap J=\{a\}$. Thus $\gamma_{S}$ is a congruence on the matroid $M_{S}$. Suppose $\gamma$ is a strong congruence on $M$. To verify that $\gamma_{S}$ also fulfills (C5), let $U \in \mathcal{I}_{S}, x \in S, B$ a basis for $U$ in the matroid $\left(S, \mathbb{I}_{\gamma_{S}}\right)$ and suppose $B \cup x \in \mathbb{I}_{\gamma_{S}}$. Then $U \in \mathcal{I}_{S} \subseteq \mathcal{I}, x \in M, B \subseteq U$ with $B \in \mathbb{I}_{\gamma_{S}} \subseteq \mathbb{I}_{\gamma}$ from which it is easy to see that $B$ is a basis for $U$ in the matroid $\left(M, \mathbb{I}_{\gamma}\right)$. Since $B \cup x \in \mathbb{I}_{\gamma_{S}} \subseteq \mathbb{I}_{\gamma}$, the validity of (C5) for the congruence $\gamma$ gives $U \cup x \in \mathcal{I}$. Then $B \cup x \in \mathcal{I}$ and hence $B \cup x=S \cap(B \cup x) \in \mathbb{I}_{\gamma_{S}}$ and the result follows.

PROPOSITION 2.12. Let $M=(E, \mathcal{I})$ and $M^{\prime}=\left(E^{\prime}, \mathcal{I}^{\prime}\right)$ be matroids with $f: M \rightarrow M^{\prime}$ a weak map, $S$ a nonempty subset of $E$ with $M_{S}=\left(S, \mathcal{I}_{S}\right)$ the associated submatroid of $M$ and $\left.f\right|_{S}: M_{S} \rightarrow M^{\prime}$ the associated weak map on the restriction of $f$ to $S$. Then $\left.\operatorname{ker} f\right|_{S}=S \cap \operatorname{ker} f$.

Proof. By Proposition 1.4 we know $\left.f\right|_{S}$ is a weak map. Let $\operatorname{ker} f=\left(\sim f, \mathbb{I}_{f}\right)$. To simplify notation, let us write $\operatorname{ker} f=\gamma=\left(\sim \gamma, \mathbb{I}_{\gamma}\right)\left(\right.$ so $\sim f=\sim \gamma$ and $\left.\mathbb{I}_{f}=\mathbb{I}_{\gamma}\right)$. We show $\left.\operatorname{ker} f\right|_{S}=\gamma_{S}$ where $\gamma_{S}=S \cap \operatorname{ker} f=$ $\left(\sim_{\gamma_{S}}, \mathbb{I}_{\gamma_{S}}\right)$ as defined above. Let $\left.\operatorname{ker} f\right|_{S}=\left(\left.\sim f\right|_{S}, \mathbb{I}_{\left.f\right|_{S}}\right)$. For $a, b \in S, a \sim_{\left.f\right|_{S}} b \Leftrightarrow f(a)=f(b) \Longleftrightarrow a \sim_{\gamma_{S}} b$. Let $I \in \mathbb{I}_{\left.f\right|_{S}}$. Then $I \subseteq S$ and $\left.f\right|_{S}: I \rightarrow f(I)$ is injective with $f(I) \in \mathcal{I}^{\prime}$, i.e., $f: I \rightarrow f(I)$ is injective. Thus $I \in \mathbb{I}_{f}=\mathbb{I}_{\gamma}$ and so $I=S \cap I \in \mathbb{I}_{\gamma_{S}}$. Conversely, let $I \in \mathbb{I}_{\gamma_{S}}$, say $I=S \cap J$ with $J \in \mathbb{I}_{\gamma}=\mathbb{I}_{f}$. Then $\left.f\right|_{J}: J \rightarrow f(J)$ is injective with $f(J) \in \mathcal{I}^{\prime}$. This gives $\left.f\right|_{S}: I \rightarrow f(I)$ injective with $f(I) \subseteq f(J) \in \mathcal{I}^{\prime}$; hence $I \in \mathbb{I}_{f \mid S}$.

For a congruence $\gamma$ on a matroid $M$ and $M_{S}$ a submatroid of $M$, if $\pi: M \rightarrow M / \gamma$ is the canonical quotient map, then $\pi(S)$ is a nonempty subset of $E / \gamma$, hence it determines a submatroid $(M / \gamma)_{\pi(S)}:=$ $\left(\pi(S),(\mathcal{I} / \gamma)_{\pi(S)}\right)$ of $M / \gamma$. This submatroid of $M / \gamma$ is denoted by $(S+\gamma) / \gamma$. Of course, the use of the symbol + here has got no relevance to any addition, or for that matter, anything else that we are presenting here, except that it shows the connection of the next result with its universal algebra counterpart.

THEOREM 2.13 (Second Isomorphism Theorem). Let $\gamma=\left(\sim_{\gamma}, \mathbb{I}_{\gamma}\right)$ be a congruence on the matroid $M=(E, \mathcal{I})$ with $M_{S}=\left(S, \mathcal{I}_{S}\right)$ a submatroid of $M$ and $S \cap \gamma=\gamma_{S}=\left(\sim \gamma_{S}, \mathbb{I}_{\gamma_{S}}\right)$ the restriction of $\gamma$ to $M_{S}$. Let $(S+\gamma) / \gamma$ be the submatroid of $M / \gamma$ determined by $\pi(S)$ where $\pi: M \rightarrow M / \gamma$ is the quotient map. Then $(S+\gamma) / \gamma \cong M_{S} / S \cap \gamma$ where $M_{S} / S \cap \gamma$ is the quotient matroid determined by the congruence $S \cap \gamma$ on the matroid $M_{S}$.

Proof. We know the quotient map $\pi: M \rightarrow M / \gamma$ has ker $\pi=\gamma$. Hence the restriction $\left.\pi\right|_{S}: M_{S} \rightarrow$ $\pi(S)=(S+\gamma) / \gamma$ is a surjective weak map with ker $\left.\pi\right|_{S}=S \cap \gamma$ by Proposition 2.12. The First Isomorphism Theorem gives the required isomorphism $M_{S} / S \cap \gamma \cong(S+\gamma) / \gamma$.

For a congruence $\alpha$ on a matroid $M$, we now describe the congruences on the quotient matroid $M / \alpha$. Let $\alpha=\left(\sim \alpha, \mathbb{I}_{\alpha}\right)$ and $\beta=\left(\sim \beta, \mathbb{I}_{\beta}\right)$ be congruences on the matroid $M$ with $\alpha \subseteq \beta$. A quotient congruence $\beta / \alpha=\left(\sim \beta / \alpha, \mathbb{I}_{\beta / \alpha}\right)$ on the matroid $M / \alpha$ is defined as follows: For $[a]_{\alpha},[b]_{\alpha} \in M / \alpha,[a]_{\alpha} \sim \beta / \alpha$ 
$[b]_{\alpha} \Leftrightarrow a \sim \beta b$ and $\mathbb{I}_{\beta / \alpha}=\left\{\pi_{\alpha}(I) \mid I \in \mathbb{I}_{\beta}\right\}$ where $\pi_{\alpha}: M \rightarrow M / \alpha$ is the canonical quotient map. That this is indeed a congruence, is part of the next result.

PROPOSITION 2.14. Let $\alpha$ and $\beta$ be congruences on the matroid $M=(E, \mathcal{I})$ with $\alpha \sqsubseteq \beta$. Then $\beta / \alpha$ is a congruence on the matroid $M / \alpha$. Conversely, if $\alpha$ is a congruence on $M$, then any congruence $\gamma$ on $M / \alpha$ is of the form $\gamma=\beta / \alpha$ for some congruence $\beta$ on $M$ with $\alpha \sqsubseteq \beta$.

Proof. Let $\alpha=\left(\sim \alpha, \mathbb{I}_{\alpha}\right)$ and $\beta=\left(\sim \beta, \mathbb{I}_{\beta}\right)$ with $\alpha \subseteq \beta$ and let $\beta / \alpha=\left(\sim \beta / \alpha, \mathbb{I}_{\beta / \alpha}\right)$. Firstly we show $\sim \beta / \alpha$ is well-defined. Let $[a]_{\alpha},\left[a^{\prime}\right]_{\alpha},[b]_{\alpha},\left[b^{\prime}\right]_{\alpha} \in M / \alpha$ with $[a]_{\alpha}=\left[a^{\prime}\right]_{\alpha}$ and $[b]_{\alpha}=\left[b^{\prime}\right]_{\alpha}$. Then $a \sim_{\alpha} a^{\prime}$ and $b \sim_{\alpha} b^{\prime}$. Since $\alpha \subseteq \beta$, we have $a \sim_{\beta} a^{\prime}$ and $b \sim_{\beta} b^{\prime}$. Thus $[a]_{\alpha} \sim \beta / \alpha[b]_{\alpha} \Leftrightarrow a \sim \beta$ $b \Leftrightarrow a^{\prime} \sim \beta b^{\prime} \Leftrightarrow\left[a^{\prime}\right]_{\alpha} \sim \beta / \alpha\left[b^{\prime}\right]_{\alpha}$. Then $(\mathrm{C} 1)$, requiring $\sim \beta / \alpha$ an equivalence relation on $M / \alpha$, easily follows. For $(\mathrm{C} 2)$, we know that $M_{\beta}=\left(E, \mathbb{I}_{\beta}\right)$ is a matroid and by Proposition 2.1, for the map $\pi_{\alpha}: E \rightarrow E / \alpha$, we have $\left(E / \alpha, \mathbb{I}_{\beta / \alpha}\right)$ is a matroid since $\mathbb{I}_{\beta / \alpha}=\pi_{\alpha}\left(\mathbb{I}_{\beta}\right)$. We also need $\mathbb{I}_{\beta / \alpha} \subseteq \mathcal{I} / \alpha$. Let $W \in \mathbb{I}_{\beta / \alpha}$. Then $W=\pi_{\alpha}(I)$ for some $I \in \mathbb{I}_{\beta}$. Since $\alpha \sqsubseteq \beta, I \in \mathbb{I}_{\alpha}$ and $W \in \mathcal{I} / \alpha$ follows. For (C3), let $A=\left\{\left[a_{1}\right]_{\alpha}, \ldots,\left[a_{n}\right]_{\alpha}\right\} \in \mathbb{I}_{\beta / \alpha}$ and $B=\left\{\left[b_{1}\right]_{\alpha}, \ldots,\left[b_{n}\right]_{\alpha}\right\} \subseteq M / \alpha$ with $|A|=|B|=n$ and $\left[a_{i}\right]_{\alpha} \sim \beta / \alpha\left[b_{i}\right]_{\alpha}$, i.e., $a_{i} \sim \beta \quad b_{i}$ for all $i=1,2, \ldots, n$. By definition of $\mathbb{I}_{\beta / \alpha}, A=\pi_{\alpha}(I)$ for some $I=\left\{a_{1}^{\prime}, \ldots, a_{n}^{\prime}\right\} \in \mathbb{I}_{\beta}$ with $\left[a_{i}\right]_{\alpha}=\pi_{\alpha}\left(a_{i}\right)=\pi_{\alpha}\left(a_{i}^{\prime}\right)=\left[a_{i}^{\prime}\right]_{\alpha}$. Thus $a_{i} \sim_{\alpha} a_{i}^{\prime}$ which gives $a_{i} \sim \beta$ and hence $a_{i}^{\prime} \sim \beta b_{i}$ for all $i$. The Substitution Property on $\beta$ ensures that $J \in \mathbb{I}_{\beta}$ and then $B=\left\{\left[b_{1}\right]_{\alpha}, \ldots,\left[b_{n}\right]_{\alpha}\right\}=$ $\left\{\pi_{\alpha}\left(b_{1}\right), \ldots, \pi_{\alpha}\left(b_{n}\right)\right\}=\pi_{\alpha}(J) \in \mathbb{I}_{\beta / \alpha}$. To conclude the proof that $\beta / \alpha$ is a congruence, we show the validity of (C4). Let $[a]_{\alpha} \in I \in \mathbb{I}_{\beta / \alpha}$, say $I=\pi_{\alpha}(V), V \in \mathbb{I}_{\beta}$ with $\pi_{\alpha}(a)=[a]_{\alpha}=\pi_{\alpha}(v)$ for some $v \in V$. Let $[b]_{\alpha} \in\left[[a]_{\alpha}\right]_{\beta / \alpha} \cap I$. Then $[a]_{\alpha} \sim_{\beta / \alpha}[b]_{\alpha}$, i.e., $a \sim_{\beta} b$ and $\pi_{\alpha}(b)=[b]_{\alpha}=\pi_{\alpha}(u)$ for some $u \in V$. We need to show $[b]_{\alpha}=[a]_{\alpha}$. From $a \sim_{\alpha} v$ and $b \sim_{\alpha} u$ we get $a \sim_{\beta} v$ and $b \sim_{\beta} u$. Since $a \sim_{\beta} b$, $u \sim \beta v$ follows. Thus $u \in[v]_{\beta} \cap V=\{v\}$, i.e., $u=v$ and then also $[b]_{\alpha}=[a]_{\alpha}$.

For the converse, let $\gamma=\left(\sim \gamma, \mathbb{I}_{\gamma}\right)$ be a congruence on the matroid $M / \alpha$. A congruence $\beta=\left(\sim \beta, \mathbb{I}_{\beta}\right)$ on $M$ is defined by: For $a, b \in M, a \sim \beta b \Leftrightarrow[a]_{\alpha} \sim_{\gamma}[b]_{\alpha}$ and $\mathbb{I}_{\beta}=\left\{I \in \mathbb{I}_{\alpha} \mid \pi_{\alpha}(I) \in \mathbb{I}_{\gamma}\right)$. That $\sim_{\beta}$ is an equivalence relation is obvious and by definition $\mathbb{I}_{\beta} \subseteq \mathbb{I}_{\alpha} \subseteq \mathcal{I}$. Requirements (M1) and (M2) for showing that $\left(E, \mathbb{I}_{\beta}\right)$ is a matroid are clear, but we have to spend some time verifying (M3). Let $I, J \in \mathbb{I}_{\beta}$ with $|I|<|J|$. Then $I, J \in \mathbb{I}_{\alpha}$ and $\pi_{\alpha}(I), \pi_{\alpha}(J) \in \mathbb{I}_{\gamma}$. Since $\alpha$ is a congruence, property (C4) gives both $\pi_{\alpha}: I \rightarrow \pi_{\alpha}(I)$ injective and $\pi_{\alpha}: J \rightarrow \pi_{\alpha}(J)$ injective and so $\left|\pi_{\alpha}(I)\right|<\left|\pi_{\alpha}(J)\right|$. Hence there is $y \in \pi_{\alpha}(J)-\pi_{\alpha}(I)$ with $\pi_{\alpha}(I) \cup y \in \mathbb{I}_{\gamma}$. Now $y=\pi_{\alpha}(b)$ for some $b \in J-I$. Consequently $\pi_{\alpha}(I \cup b)=\pi_{\alpha}(I) \cup y \in \mathbb{I}_{\gamma} \subseteq \mathcal{I} / \alpha$ which means $\pi_{\alpha}(I \cup b)=\pi_{\alpha}(V)$ for $V \in \mathbb{I}_{\alpha}$. This latter membership ensures $\pi_{\alpha}: V \rightarrow \pi_{\alpha}(V)$ is injective and so is $\pi_{\alpha}: I \cup b \rightarrow \pi_{\alpha}(I \cup b)$ since $b \notin I$ and $y \notin \pi_{\alpha}(I)$. Thus $|I \cup b|=|V|$ which together with $\pi_{\alpha}(I \cup b)=\pi_{\alpha}(V)$ give $I \cup b \in \mathbb{I}_{\alpha}$ by the Substitution Property for the congruence $\alpha$. Hence $I \cup b \in \mathbb{I}_{\beta}$ and $\left(E, \mathbb{I}_{\beta}\right)$ is a matroid. We now verify condition (C4) for $\beta$. Let $a \in I \in \mathbb{I}_{\beta}$ and choose $b \in[a]_{\beta} \cap I$. Then $b \sim \beta a$ and $b \in I \in \mathbb{I}_{\alpha}$ with $\pi_{\alpha}(I) \in \mathbb{I}_{\gamma}$. The definition of $\sim \beta$ gives $[a]_{\alpha} \sim \gamma[b]_{\alpha}$ and so $[b]_{\alpha} \in\left[[a]_{\alpha}\right]_{\gamma} \cap \pi_{\alpha}(I)=\left\{[a]_{\alpha}\right\}$ since $\gamma$ is a congruence of $M / \alpha$. Then $b \sim_{\alpha} a$ and thus $b \in[a]_{\alpha} \cap I=\{a\}$ giving the desired equality $a=b$. Lastly we check the Substitution Property for $\beta$. Let $I=\left\{a_{1}, a_{2}, \ldots, a_{n}\right\} \in \mathbb{I}_{\beta}$ and $J=\left\{b_{1}, b_{2}, \ldots, b_{n}\right\} \subseteq E$ with $|I|=|J|=n$ and $a_{i} \sim \beta b_{i}$ for all $i=1,2, \ldots, n$. We know $I \in \mathbb{I}_{\alpha}$ and $\pi_{\alpha}(I) \in \mathbb{I}_{\gamma}$. Moreover, $\pi_{\alpha}: I \rightarrow \pi_{\alpha}(I)$ is injective and $a_{i} \sim \beta \quad b_{i}$ implies $\left[a_{i}\right]_{\alpha} \sim \gamma\left[b_{i}\right]_{\alpha}$ for all $i$. Also, $\left|\pi_{\alpha}(J)\right|=n$, for if $\pi_{\alpha}\left(b_{i}\right)=\pi_{\alpha}\left(b_{j}\right)$ for some $i \neq j$, then $b_{i} \sim \alpha b_{j}$ which implies $b_{i} \sim \beta \quad b_{j}$ and in turn gives $a_{i} \sim \beta a_{j}$. It was already shown that $\beta$ fulfills (C4), so we get $a_{i} \in\left[a_{j}\right]_{\beta} \cap I=\left\{a_{j}\right\}$ giving the contradiction $a_{i}=a_{j}$. Thus $\left|\pi_{\alpha}(J)\right|=n$ and by the Substitution Property for the congruence $\gamma$ we have $\pi_{\alpha}(J) \in \mathbb{I}_{\gamma} \subseteq \mathcal{I} / \alpha$. This means $\pi_{\alpha}(J)=\pi_{\alpha}(W)$ for some $W \in \mathbb{I}_{\alpha}$. The Substitution Property for the congruence $\alpha$ gives $J \in \mathbb{I}_{\alpha}$ and consequently $J \in \mathbb{I}_{\beta}$. Thus $\beta$ is a congruence on $M$.

Clearly $\alpha \subseteq \beta$ and to conclude the proof, we still need $\gamma=\beta / \alpha$. But this equality follows more-or-less immediately from the definitions of $\sim \beta, \sim \beta / \alpha, \mathbb{I}_{\beta}$ and $\mathbb{I}_{\beta / \alpha}$.

THEOREM 2.15 (Third Isomorphism Theorem). Let $\alpha$ and $\beta$ be congruences on the matroid $M=(E, \mathcal{I})$ with $\alpha \sqsubseteq \beta$. Then $\beta / \alpha$ is a congruence on the matroid $M / \alpha$ and $(M / \alpha) /(\beta / \alpha) \cong M / \beta$.

Proof. By Proposition 2.14, $\beta / \alpha$ is a congruence on $M / \alpha$. We have two surjective weak maps $\pi_{\alpha}: M \rightarrow M / \alpha$ and $\pi_{\beta}: M \rightarrow M / \beta$ with kernels $\alpha$ and $\beta$ respectively. By Proposition 2.8, there is a surjective weak map $f: M / \alpha \rightarrow M / \beta$ with $f \circ \pi_{\alpha}=\pi_{\beta}$, i.e., $f\left([a]_{\alpha}\right)=[a]_{\beta}$ for all $a \in M$. Our 
first observation here is that $\operatorname{ker} f=\beta / \alpha$. Indeed, $[a]_{\alpha} \sim f[b]_{\alpha} \Leftrightarrow f\left([a]_{\alpha}\right)=f\left([b]_{\alpha}\right) \Longleftrightarrow[a]_{\beta}=$ $[b]_{\beta} \Leftrightarrow a \sim \beta b \Leftrightarrow[a]_{\alpha} \sim \beta / \alpha[b]_{\alpha}$. Let $I \in \mathbb{I}_{f}$. Then $I \subseteq M / \alpha$ and $f: I \rightarrow f(I)$ is injective with $f(I) \in \mathcal{I} / \beta$. Now $I=\pi_{\alpha}(V)$ for some $V \subseteq M$ and $\pi_{\beta}(V)=f\left(\pi_{\alpha}(V)\right)=f(I)=\pi_{\beta}(W)$ for some $W \in \mathbb{I}_{\beta}$, this last equality by the definition of $\mathcal{I} / \beta$. Without loss of generality, we can choose both $V$ and $W$ minimal as subsets of $E$, i.e., both $\pi_{\alpha}: V \rightarrow \pi_{\alpha}(V)=I$ and $\pi_{\beta}: W \rightarrow \pi_{\beta}(W)=f(I)$ are injective. This gives $|V|=|W|$ and from $\pi_{\beta}(V)=\pi_{\beta}(W)$, the Substitution Property of $\beta$ gives $V \in \mathbb{I}_{\beta}$. Thus $I=\pi_{\alpha}(V) \in \mathbb{I}_{\beta / \alpha}$. Conversely, if $I \in \mathbb{I}_{\beta / \alpha}$, then $I=\pi_{\alpha}(U)$ for some $U \in \mathbb{I}_{\beta}$. Now $\pi_{\beta}: U \rightarrow \pi_{\beta}(U)$ is injective, and hence $f: \pi_{\alpha}(U) \rightarrow f\left(\pi_{\alpha}(U)\right)=\pi_{\beta}(U) \in \mathcal{I} / \beta$ is injective. This means $I=\pi_{\alpha}(U)$ is in $\mathbb{I}_{f}$ giving the equality $\mathbb{I}_{f}=\mathbb{I}_{\beta / \alpha}$ and hence $\operatorname{ker} f=\beta / \alpha$. An application of the First Isomorphism Theorem gives $(M / \alpha) /(\beta / \alpha)=(M / \alpha) / \operatorname{ker} f \cong M / \beta$.

COROLLARY 2.16. Let $\alpha$ be a congruences on a matroid $M$. Then there is a one-to-one correspondence between the set $\{\beta \mid \beta$ is a congruence on $M$ with $\alpha \sqsubseteq \beta\}$ and the set $\{\gamma \mid \gamma$ is a congruence on $M / \alpha\}$. This correspondence preserves inclusions.

This concludes our definition and first results for a theory of congruences on matroids. In the last two sections we look at some applications.

\section{SUBDIRECT PRODUCTS AND BIRKHOFF'S THEOREM}

The notion of a (cartesian) product of matroids is not in a natural way compatible with the axioms defining a matroid. It is well-known that the category of matroids does not have products. It is probably for this reason that the construction of subdirect products for matroids has not been considered. A subdirect product is a substructure of the product that sits tightly in the product in some sense and is an important construction in algebra, but it has also been used in topology and graph theory albeit under different guises. The notion of a subdirect product gives rise to subdirectly irreducible objects and then, in many cases, is followed by a result stating that any nontrivial object is a subdirect product of subdirectly irreducible objects (Birkhoff's Theorem). In this section it will be shown that it is possible to define a subdirect product for matroids, talk about subdirectly irreducible matroids and then formulate and proof a version of Birkhoff's Theorem for matroids.

For background, we should mention two matroid constructions. For matroids $M_{1}, M_{2}, \ldots, M_{n}$, $M_{j}=\left(E_{j}, \mathcal{I}_{j}\right.$ ) for all $j$, the union (or sum) $M=(E, \mathcal{I})$, written as $M=M_{1} \vee M_{2} \vee \cdots \vee M_{n}$, is the matroid with $E=E_{1} \cup E_{2} \cup \cdots \cup E_{n}$ and $\mathcal{I}=\left\{I_{1} \cup I_{2} \cup \cdots \cup I_{n} \mid I_{j} \in \mathcal{I}_{j}, j=1,2,3, \ldots, n\right\}$. We will write this latter set consisting of the union of independent subsets as $\mathcal{I}=\mathcal{I}_{1} \vee \mathcal{I}_{2} \vee \cdots \vee \mathcal{I}_{n}$. This should not be confused with $\mathcal{I}_{1} \cup \mathcal{I}_{2} \cup \cdots \cup \mathcal{I}_{n}=\left\{I \subseteq M \mid I \in \mathcal{I}_{j}\right.$ for some $\left.1 \leq j \leq n\right\}$ which, in general, need not give a matroid on $E$. If all the ground sets $E_{j}$ are pairwise disjoint, then $M$ is called the direct sum and is denoted by $M=M_{1} \oplus M_{2} \oplus \cdots \oplus M_{n}$. A matroid $M$ is reducible if it can be written as anion of two matroids, neither of which is equal to $M$; otherwise it is called irreducible. Below we will define the different notion of a subdirectly irreducible matroid.

DEFINITION 3.1. A matroid $S=(E, \mathcal{I})$ is a subdirect product of matroids $M_{1}=\left(E_{1}, \mathcal{I}_{1}\right), M_{2}=\left(E_{2}, \mathcal{I}_{2}\right)$, $\ldots, M_{n}=\left(E_{n}, \mathcal{I}_{n}\right)$ if for every $j=1,2, \ldots, n$, there is a surjective weak map $p_{j}: S \rightarrow M_{j}$ such that setting $\left(\sim j, \mathbb{I}_{j}\right):=\operatorname{ker} p_{j}$ for all $j \leq n$, the following hold:

(1) For $a, b \in S$, if $a \sim_{j} b$ for all $j \leq n$, then $a=b$; and

(2) $\mathcal{I} \subseteq \mathbb{I}_{1} \vee \mathbb{I}_{2} \vee \cdots \vee \mathbb{I}_{n}$.

With the notation as in Definition 3.1 and setting $\alpha_{j}:=\operatorname{ker} p_{j}$, we know $M_{j} \cong S / \alpha_{j}$ for all $j \leq n$ by the First Isomorphism Theorem; so $S$ is actually a subdirect product of the $S / \alpha_{j}$ 's. A matroid $M$ is a subdirect product of one matroid $M_{1}$ if and only if $M \cong M_{1}$. For sets $E_{1}, E_{2}, \ldots, E_{n}$, let $E_{1} \times E_{2} \times \cdots \times E_{n}$ be their usual cartesian product with $\pi_{j}: E_{1} \times E_{2} \times \cdots \times E_{n} \rightarrow E_{j}$ the $j$-th projection. In some sense, the next result motivates the use of the terminology subdirect product.

PROPOSITION 3.2. Let $S=(E, \mathcal{I})$ be a subdirect product of the matroids $M_{1}=\left(E_{1}, \mathcal{I}_{1}\right), M_{2}=\left(E_{2}, \mathcal{I}_{2}\right)$, $\ldots, M_{n}=\left(E_{n}, \mathcal{I}_{n}\right)$ with surjective weak maps $p_{j}: S \rightarrow M_{j}$ for all $j$. Then there is a function $g: E \rightarrow E_{1} \times E_{2} \times \cdots \times E_{n}$ such that $M=(g(E), g(\mathcal{I}))$ is a matroid isomorphic to $S$ and $\pi_{j}^{\prime} \circ g=p_{j}$ for all $j$ where $\pi_{j}^{\prime}: M \rightarrow M_{j}$ is the restriction of $\pi_{j}$ to $g(E)$. Each $\pi_{j}^{\prime}$ is a surjective weak map. 
Proof. Define $g: E \rightarrow E_{1} \times E_{2} \times \cdots \times E_{n}$ by $g(s)=\left(p_{1}(s), p_{2}(s), \ldots, p_{n}(s)\right)$ for all $s \in E$. This is a well-defined function and by Proposition 2.1, $M:=(g(E), g(\mathcal{I}))$ is a matroid. Suppose $g(a)=g(b)$ for some $a, b \in E$. Then $p_{j}(a)=p_{j}(b)$, i.e., $a \sim_{j} b$ for all $j=1,2, \ldots, n$ and by the definition of a subdirect product, $a=b$. Thus $g: E \rightarrow g(E)$ is a bijection and clearly $\pi_{j}^{\prime} \circ g=p_{j}$ for all $j$. Since $p_{j}$ is surjective, so is $\pi_{j}^{\prime}$. Next we verify that $g$ is a weak map: Let $J \subseteq E$ such that $g: J \rightarrow g(J)$ is injective with $g(J) \in g(\mathcal{I})$, say $g(J)=g(I)$ for some $I \in \mathcal{I}$. By the injectivity of $g, J=I \in \mathcal{I}$ holds. Also, $g^{-1}$ is a weak map: Let $I \subseteq g(E)$ such that $g^{-1}: I \rightarrow g^{-1}(I) \in \mathcal{I}$ is injective. Now $I=g\left(g^{-1}(I)\right) \in g(\mathcal{I})$. It follows that $g$ is an isomorphism (by Proposition 1.3(1)). Lastly it is shown that $\pi_{j}^{\prime}: M \rightarrow M_{j}$ is a weak map. Let $I \subseteq g(E)$ such that $\pi_{j}^{\prime}: I \rightarrow \pi_{j}^{\prime}(I) \in \mathcal{I}_{j}$ is injective. Now $I=g(U)$ for some $U \subseteq E$ and $p_{j}: U \rightarrow p_{j}(U)=\pi_{j}^{\prime}(I) \in \mathcal{I}_{j}$ is injective. Since $p_{j}: S \rightarrow M_{j}$ is a weak map, $U \in \mathcal{I}$ and hence $I \in g(\mathcal{I})$ follows.

We now give an example of a subdirect product; more examples will follow below. This example can be given in a more general setting, but the special case below suffices to convey the basic ideas.

EXAMPLE 3.3. Let $E$ be a nonempty finite subset of $\mathbb{R}^{5}$ with $\mathcal{I}=\{A \subseteq E \mid A=\varnothing$ or $A$ is a linearly independent subset of $E\}$. Then $M:=(E, \mathcal{I})$ is a matroid. Let $p_{1}: E \rightarrow \mathbb{R}^{3}$ and $p_{2}: E \rightarrow \mathbb{R}^{2}$ be the projection maps $p_{1}\left(a_{1}, a_{2}, a_{3}, a_{4}, a_{5}\right)=\left(a_{1}, a_{2}, a_{3}\right)$ and $p_{2}\left(a_{1}, a_{2}, a_{3}, a_{4}, a_{5}\right)=\left(a_{4}, a_{5}\right)$ for all $\left(a_{1}, a_{2}, a_{3}, a_{4}, a_{5}\right) \in E$. For $j=1,2, M_{j}:=\left(p_{j}(E), \mathcal{I}_{j}\right)$ is a matroid where $\mathcal{I}_{j}:=\left\{A \subseteq p_{j}(E) \mid A=\varnothing\right.$ or $A$ is a linearly independent subset of $\left.p_{j}(E)\right\}$. Then $M$ is a subdirect product of $M_{1}$ and $M_{2}$ : Each $p_{j}: M \rightarrow M_{j}$ is a surjective weak map. Let $\operatorname{ker} p_{j}=\alpha_{j}=\left(\sim j, \mathbb{I}_{j}\right)$. Clearly, for $a, b \in E, p_{j}(a)=p_{j}(b)$ for $j=1,2$ implies $a=b$. To show that $\mathcal{I} \subseteq \mathbb{I}_{1} \vee \mathbb{I}_{2}$, we use the following result, probably well known but I could not find a reference to it or actually to a more general formulation.

Let $A=\left\{v_{1}, v_{2}, \ldots, v_{5}\right\}$ be a linearly independent subset of $\mathbb{R}^{5}$, say $v_{j}=\left(v_{1 j}, v_{2 j}, v_{3 j}, v_{4 j}, v_{5 j}\right)^{T}$. Let $V=\left[v_{i j}\right]$ be the $5 \times 5$ matrix with columns the vectors $v_{j}$. Since the vectors $v_{1}, v_{2}, \ldots, v_{5}$ are linearly independent, $\operatorname{det}(V) \neq 0$. Using a cofactor expansion along the fifth row and then along the fourth row in each of the five $4 \times 4$ minors, we get

$$
\operatorname{det}(v)=\sum_{i=1}^{3} \sum_{j=i+1}^{4} \sum_{k=j+1}^{5}(-1)^{i+j+k}\left|\begin{array}{lll}
v_{1 i} & v_{1 j} & v_{1 k} \\
v_{2 i} & v_{2 j} & v_{2 k} \\
v_{3 i} & v_{3 j} & v_{3 k}
\end{array}\right| \begin{array}{ll}
v_{4 s} & v_{4 t} \\
v_{5 s} & v_{5 t}
\end{array} \mid,
$$

where $s, t \in\{1,2,3,4,5\}-\{i, j, k\}$ with $s<t$. Since $\operatorname{det}(V) \neq 0$, there must be at least one choice of $i, j$ and $k$ such that

$$
\left|\begin{array}{lll}
v_{1 i} & v_{1 j} & v_{1 k} \\
v_{2 i} & v_{2 j} & v_{2 k} \\
v_{3 i} & v_{3 j} & v_{3 k}
\end{array}\right|\left|\begin{array}{ll}
v_{4 s} & v_{4 t} \\
v_{5 s} & v_{5 t}
\end{array}\right| \neq 0
$$

This means $\left\{\left(v_{1 i}, v_{2 i}, v_{3 i}\right),\left(v_{1 j}, v_{2 j}, v_{3 j}\right),\left(v_{1 k}, v_{2 k}, v_{3 k}\right)\right\}$ and $\left\{\left(v_{4 s}, v_{5 s}\right),\left(v_{4 t}, v_{5 t}\right)\right\}$ are linearly independent sets in $\mathbb{R}^{3}$ and $\mathbb{R}^{2}$ respectively.

It is now shown that $\mathcal{I} \subseteq \mathbb{I}_{1} \vee \mathbb{I}_{2}$. Let $\varnothing \neq B \in \mathcal{I}$. From the result above, $B=B_{1} \cup B_{2}$ for two nonempty disjoint subsets $B_{1}, B_{2}$ of $B$ with $p_{j}\left(B_{j}\right)$ linearly independent in $M_{j}$ for $j=1,2$. Then $p_{j}: B_{j} \rightarrow p_{j}\left(B_{j}\right) \in \mathcal{I}_{j}$ is an injective map for both values of $j$ and hence $B_{1} \in \mathbb{I}_{1}$ and $B_{2} \in \mathbb{I}_{2}$. Thus $\mathcal{I} \subseteq \mathbb{I}_{1} \cup \mathbb{I}_{2}$.

A matroid $M$ is subdirectly irreducible if it satisfies the following condition: whenever $M$ is a subdirect product of matroids $M_{1}, M_{2}, \ldots, M_{n}$, then $M \cong M_{i}$ for some $i$. If $M$ is not subdirectly irreducible, then it is called subdirectly reducible.

EXAMPLE 3.4. Let $E=\{a, b\}$ be a set with two distinct elements. Let $\mathcal{I}=\{\varnothing,\{a\}\}$. We show $M:=$ $(E, \mathcal{I})$ is a subdirectly irreducible matroid. Clearly $M$ is a matroid. There are only two equivalence relations possible on $E$ namely $\approx$ and $\leadsto$ and only two possible congruence independent sets $\mathbb{I}_{1}=\{\varnothing\}$ and $\mathbb{I}_{2}=\{\{\varnothing\},\{a\}\}=\mathcal{I}$. This means there are precisely three congruences on $M$, namely $\iota_{M}=(\approx, \mathcal{I}), \tau=(\approx,\{\varnothing\})$ and $v_{M}=(\rightsquigarrow,\{\varnothing\})$. Suppose $M$ is a subdirect product of two matroids $M_{1}$ and $M_{2}$ with associated surjective weak maps $p_{j}: M \rightarrow M_{j}$ and $\alpha_{i}=\operatorname{ker} p_{j}=\left(\sim j, \mathbb{I}_{j}\right)$. We know $M_{j} \cong M / \alpha_{j}$ and $\alpha_{1}, \alpha_{2} \in\left\{\iota_{M}, \tau, v_{M}\right\}$ and need to show $M / \alpha_{j} \cong M$ for at least one $j$. If any $\alpha_{j}=\iota_{M}$, we are done, so suppose $\alpha_{1}, \alpha_{2} \in\left\{\tau, v_{M}\right\}$. We also know $\sim_{1} \cap \sim_{2}=\approx$, so the only possibilities for 
the pair $\left(\alpha_{1}, \alpha_{2}\right)$ are $(\tau, \tau)$ or $\left(\tau, v_{M}\right)$. But for both these choices, we cannot have $\mathcal{I} \subseteq \mathbb{I}_{1} \vee \mathbb{I}_{2}$. Hence one of the matroids $M_{1}$ or $M_{2}$ must be isomorphic to $M$. A similar argument shows that if $M$ is a subdirect product of three or more matroids, one of these must already be isomorphic to $M$. Thus $M$ is subdirectly irreducible.

EXAMPLE 3.5. Let $E=\{a, b\}$ be a set with two distinct elements. Let $\mathcal{I}=\{\varnothing\}$. Then $M:=(E, \mathcal{I})$ is a subdirectly irreducible matroid. This follows immediately along the lines of the previous example since there are only two congruences on $M$ possible, namely $\iota_{M}$ and $v_{M}$.

EXAMPLE 3.6. Let $E=\{a, b\}$ be a set with two distinct elements. Let $\mathcal{I}=\{\varnothing,\{a\},\{b\}\}$. Then $M:=$ $(E, \mathcal{I})$ is a matroid which is subdirectly reducible: $\gamma_{a}=(\approx,\{\varnothing,\{a\}\})$ and $\gamma_{b}=(\approx,\{\varnothing,\{b\}\}$ are congruences on $M, \iota_{M}$ is properly contained in both and $M$ is a subdirect product of $M / \gamma_{a}$ and $M / \gamma_{b}$.

The last example is a special case of Birkhoff's Theorem for matroids which is the next result.

THEOREM 3.7 (Birkhoff's Theorem for matroids). Every nontrivial matroid is a subdirect product of subdirectly irreducible matroids.

Proof. Let $M=(E, \mathcal{I})$ be a matroid with $|E|>1$. For each $a \in M$, let $\gamma_{a}=\left(\sim_{a}, \mathbb{I}_{a}\right)$ be the congruence on $M$ where $\sim a$ is the equivalence relation on $E$ which has exactly two equivalence classes namely $\{a\}$ and $E-\{a\}$ and

$$
\mathbb{I}_{a}= \begin{cases}\{\varnothing,\{a\}\} & \text { if }\{a\} \text { is independent, } \\ \{\varnothing\} & \text { if }\{a\} \text { is a loop. }\end{cases}
$$

We show $\gamma_{a}$ is a congruence when $\{a\}$ is independent; the other case being obvious. Clearly requirements (C1) and (C2) are fulfilled. For (C3), let $A \in \mathbb{I}_{a}$ and $B \subseteq M$ such that $|A|=|B|$ and elements of $A$ are pairwise equivalent to the ones in $B$. Now $A=\varnothing$ or $A=\{a\}$. If $A=\varnothing$, then $B=\varnothing \in \mathbb{I}_{a}$. If $A=\{a\}$, then $B=\{b\}$ for some $b \sim a$. This means $b=a$ and $B=A \in \mathbb{I}_{a}$. Requirement (C4), $[x] \cap A=\{x\}$ for all $x \in A \in \mathbb{I}_{a}$ holds trivially.

Next we show that $M$ is a subdirect product of the matroids $M / \gamma_{a}$ for $a \in M$. Let $p_{a}=\pi_{a}: M \rightarrow$ $M / \gamma_{a}$ be the canonical quotient map, which we know is a surjective weak map with ker $p_{a}=\gamma_{a}$ for all $a \in M$. Let $x, y \in M$ with $x \sim a y$ for all $a \in M$. In particular, this is valid for $a=x$ which then yields $x=y$. Let $I=\left\{a_{1}, a_{2}, \ldots, a_{n}\right\} \in \mathcal{I}$. By the downwards closed property of independent sets, each $\left\{a_{i}\right\}$ is independent in $M$ and hence $\mathbb{I}_{a_{i}}=\left\{\varnothing,\left\{a_{i}\right\}\right\}$. Thus $I=\left\{a_{1}\right\} \cup\left\{a_{2}\right\} \cup \cdots \cup\left\{a_{n}\right\} \in \bigvee_{a \in M} \mathbb{I}_{a}$ and so $\mathcal{I} \subseteq \bigvee_{a \in M} \mathbb{I}_{a}$. Hence $M$ is a subdirect product of the matroids $M / \gamma_{a}$ for $a \in M$.

For every $a \in M$, the matroid $M / \gamma_{a}=\left(E_{a}, \mathcal{I}_{a}\right)$ has $E_{a}=\{[a],[b]\}$ for any $b \neq a$ in $M$. Moreover,

$$
\mathcal{I}_{a}=\left\{\pi_{a}(I) \mid I \in \mathbb{I}_{a}\right\}= \begin{cases}\{\varnothing,\{[a]\}\} & \text { if }\{a\} \text { is independent, } \\ \{\varnothing\} & \text { if }\{a\} \text { is a loop. }\end{cases}
$$

For both possibilities, we know $M / \gamma_{a}$ is subdirectly irreducible (see Examples 3.4 and 3.5). We conclude that every non-trivial matroid is a subdirect product of subdirectly irreducible matroids.

As is usually the case wherever they exist, one would like to explicitly describe all the subdirectly irreducible matroids. This will not be done here. In the next section, two more examples of congruences and another subdirect product of matroids will be given.

\section{EXAMPLES OF CONGRUENCES}

In this section we discuss two well-known equivalence relations on a matroid and some of their properties. It will be seen that each gives rise to a natural congruence on a matroid and the properties of these congruences suggest that it may be possible to accommodate them within a radical theory for matroids. Radical theory has its origins in ring theory (see, for example, [4]) and group theory, but has spread to all branches of algebra (semi-groups, $\Gamma$-rings, $\mathcal{S}$-acts, etc.). It has even spread beyond algebra and was defined and developed for topological spaces [1] and graphs [3] where it is known as a theory of connectednesses and disconnectednesses. It is not our intention to investigate or develop such a theory for matroids here. 
For a matroid $M=(E, \mathcal{I})$, we know that $\mathcal{I}$ is a subset of the power set $\mathcal{P}(E)$ of $E$. The matroid $M$ determined by the independent sets in $\mathcal{I}$ is essentially independent of the ground set $E$ in the sense that if $E^{\prime}$ is any set with $\mathcal{I} \subseteq \mathcal{P}\left(E^{\prime}\right)$, then $M^{\prime}=\left(E^{\prime}, \mathcal{I}\right)$ is a matroid with the same independent subsets as $M$, the only difference being more or less or even no loops. We will, as is often done, require that all matroids under discussion in this section have no loops. This is done mainly to avoid long-winded formulations. This assumption, in particular means that $\mathcal{I}=\{\varnothing\}$ can no longer be the collection of independent sets for a matroid. In this setting, there is only one (up to isomorphism) trivial matroid $M=(\{a\},\{\varnothing,\{a\}\})$ and the universal congruence $v_{M}=(\leftrightarrow m, \mathbb{I})$ on a matroid $M=(E, \mathcal{I})$ has $\mathbb{I}=\{\varnothing\} \cup\{\{a\} \mid a \in E\}$. We still have, for any congruence $\gamma$ on $M, \gamma \subseteq v_{M}$. Moreover, under this assumption, any two-element dependent subset is a circuit. If $M$ is a matroid with no loops, then the submatroid $M_{S}=\left(S, \mathcal{I}_{S}\right)$ will not have loops. Furthermore, when dealing with a congruence $\gamma=\left(\sim \gamma, \mathbb{I}_{\gamma}\right)$, the matroid $M_{\gamma}=\left(E, \mathbb{I}_{\gamma}\right)$ should also have no loops. In such a case, the quotient $M / \gamma_{M}$ will not have any loops.

\subsection{Simple matroids}

On a matroid $M=(E, \mathcal{I})$, two elements $x, y \in M$ are called parallel if they are related as follows: $x \sim \rho y \Leftrightarrow x=y$ or $\{x, y\}$ is a circuit. Let $\rho_{M}:=\left(\sim \rho, \mathbb{I}_{\rho}\right)$ where $\mathbb{I}_{\rho}=\{I \in \mathcal{I} \mid$ for all $a \in I,[a] \cap I=\{a\}\}$. PROPOSITION 4.1. For a matroid $M=(E, \mathcal{I}), \rho_{M}:=\left(\sim \rho, \mathbb{I}_{\rho}\right)$ is a strong congruence on $M$.

Proof. For $(\mathrm{C} 1)$ we need $\sim \rho$ an equivalence relation on $E$. But this is already a well-known equivalence relation and we only show the transitivity for completeness. Suppose $x \sim \rho y$ and $y \sim \rho z$ with $x \neq z$. Then $C_{1}=\{x, y\}$ and $C_{2}=\{y, z\}$ are circuits and since $y \in C_{1} \cap C_{2}$, the circuit axioms provide a circuit $C_{3}$ contained in $C_{1} \cap C_{2}-y=\{x, z\}$. Thus $C_{3}=\{x, z\}$ and $x \sim \rho z$.

(C2) By definition $\mathbb{I}_{\rho} \subseteq \mathcal{I}$ and the conditions (M1) and (M2) for showing $M_{\rho}=\left(E, \mathbb{I}_{\rho}\right)$ is a matroid are obvious. In order to show the validity of the Independence augmentation axiom, we first show that the Substitution Property holds.

(C3) Let $A=\left\{a_{1}, a_{2}, \ldots, a_{n}\right\} \in \mathbb{I}_{\rho}, B=\left\{b_{1}, b_{2}, \ldots, b_{n}\right\} \subseteq M$ with $|A|=|B|=n$ and $a_{i} \sim \rho b_{i}$ for all $i=1,2, \ldots, n$. Since $A \in \mathbb{I}_{\rho}$, we know that $a_{i}$ is not related to $a_{j}$ for any $i \neq j$ which in turn means that $b_{i}$ is not related to $b_{j}$ for $i \neq j$ (since $a_{i} \sim \rho b_{i}$ for all $i$ ). Thus $\left[b_{i}\right] \cap B=\left\{b_{i}\right\}$ for all $i$ and all $n$. For $B \in \mathbb{I}_{\rho}$, we still need $B \in \mathcal{I}$ and for this we will proceed by induction on $n$. For $n=1$, since any one-element subset is an independent set, $B=\left\{b_{1}\right\} \in \mathcal{I}$. For $n=2$, if $B=\left\{b_{1}, b_{2}\right\}$ is dependent, then it is a circuit which gives the contradiction $b_{1} \sim_{\rho} b_{2}$. Thus $B$ is independent and hence in $\mathcal{I}$. Let $k \geq 2$ and suppose the statement is true for all positive integers $n \leq k$. Let $n=k+1$ and suppose $A=\left\{a_{1}, a_{2}, \ldots, a_{k+1}\right\} \in \mathbb{I}_{\rho}, B=\left\{b_{1}, b_{2}, \ldots, b_{k+1}\right\} \subseteq M$ with $|A|=|B|=k+1$ and $a_{i} \sim \rho b_{i}$ for all $i=1,2, \ldots, k+1$. By the induction assumption, $B_{1}:=\left\{b_{1}, b_{2}, \ldots, b_{k-1}, b_{k+1}\right\} \in \mathbb{I}_{\rho} \subseteq \mathcal{I}$ and since $A \in \mathbb{I}_{\rho} \subseteq \mathcal{I}$, there is an $x \in A-B_{1}$ with $B_{2}:=B_{1} \cup x \in \mathcal{I}$. This $x$ must be $a_{k}$, for if $x=a_{i}$ for $i \neq k$, then $a_{i} \neq b_{i}$ and since $a_{i} \sim \rho b_{i}$, the circuit $\left\{a_{i}, b_{i}\right\}$ is contained in the independent set $B_{2}$; clearly not possible. Thus $B_{2}:=\left\{b_{1}, b_{2}, \ldots, b_{k-1}, a_{k}, b_{k+1}\right\} \in \mathcal{I}$. Again, by the induction assumption, $B_{3}:=\left\{b_{1}, b_{2}, \ldots, b_{k}\right\} \in \mathbb{I}_{\rho} \subseteq \mathcal{I}$ and there is a $y \in B_{2}-B_{3}$ with $B_{3} \cup y \in \mathcal{I}$. As in the previous case, the only possibility for $y$ is $b_{k+1}$ and we can conclude that $B=B_{3} \cup y \in \mathcal{I}$.

(M3) We now complete the proof that $M_{\rho}=\left(E, \mathbb{I}_{\rho}\right)$ is a matroid. Let $A, B \in \mathbb{I}_{\rho}$ with $|A|<|B|$. We know that no two elements of $A$ are related and likewise for $B$. Choose $A^{\prime} \subseteq A$ maximal with respect to every element of $A^{\prime}$ is related to an element of $B$. Let $B^{\prime}$ be the corresponding subset of $B$. We know $\left|A^{\prime}\right|=\left|B^{\prime}\right|$ and so $B^{\prime \prime}:=\left(B-B^{\prime}\right) \cup A^{\prime}$ has the same cardinality as $B$. Moreover, by the Substitution Property, $B^{\prime \prime} \in \mathbb{I}_{\rho}$. Then there is an element $b \in B^{\prime \prime}-A$ such that $A \cup b \in \mathcal{I}$. Not only is $b$ not in $A$, it is not even related to any element in $A$. Next we show $A \cup b \in \mathbb{I}_{\rho}$. Let $x \in A \cup b$ and choose $y \in[x] \cap(A \cup b)$. We need $y=x$ which is obvious if $x=b=y$, so there are three more cases to consider. If both $x$ and $y$ are in $A \in \mathbb{I}_{\rho}, y \in[x] \cap A=\{x\}$ gives $x=y$. The remaining two cases are not possible. Indeed, if $x \in A$ and $y=b$, then $b=y \sim \rho x \in A$ contradicting the choice of $b$; likewise $y \in A$ and $x=b$ is not possible.

(C4) This requirement is given by the definition of $\mathbb{I}_{\rho}$. 
(C5) Let $U$ be an independent subset of $M, B$ a basis for $U$ in the matroid $M_{\rho}=\left(E, \mathbb{I}_{\rho}\right)$ and $x \in M$ with $[x] \cap U=\varnothing$. Suppose $B \cup x \in \mathbb{I}_{\rho}$. We need to show that $U \cup x \in \mathcal{I}$ which will follow if $U=B$ which, in turn, will follow if we can show $U \in \mathbb{I}_{\rho}$. And for this we only need $[a] \cap U=\{a\}$ for any $a \in U$. Let $b \in[a] \cap U$. The equivalence $b \sim \rho a$ means $b=a$ or $\{a, b\}$ a circuit. But the latter is not possible since $\{a, b\} \subseteq U \in \mathcal{I}$.

Recall, a simple matroid is a matroid which has no loops and no 2-circuits. Already by the assumption at the beginning of this section, all matroids considered here are without loops. A matroid $M$ is totally disconnected if $M$ has no circuits. This is equivalent to requiring every subset of $M$ to be independent. Clearly, every totally disconnected matroid is simple. Some of the properties of the congruence $\rho_{M}$ are given in the next two results. Remember, under the assumption of no loops, the universal congruence on a matroid $M=(E, \mathcal{I})$ is $v_{M}=(\rightsquigarrow, \mathbb{I})$ where $\mathbb{I}=\{\varnothing\} \cup\{\{a\} \mid a \in E\}$. Also recall from Section 2, for a congruence $\gamma$ on a matroid $M, \gamma=v_{M} \Leftrightarrow M / \gamma$ is trivial and $\gamma=\iota_{M} \Longleftrightarrow M / \gamma \cong M$.

PROPOSITION 4.2. For a matroid $M=(E, \mathcal{I})$, let $\rho_{M}:=\left(\sim \rho, \mathbb{I}_{\rho}\right)$ be the congruence as defined above. Then:

(1) The quotient matroid $M / \rho_{M}$ is simple.

(2) $\rho_{M}=v_{M}$ (or equivalently, $M / \rho_{M}$ is trivial) if and only if all pairs of elements of $M$ are parallel.

Proof. (1) Let $\{[a],[b]\}$ be a 2-circuit in $M / \rho_{M}$. Then $a$ is not parallel to $b$; hence $a \neq b$ and $I:=\{a, b\}$ is independent. Thus $I \in \mathbb{I}_{\rho}$ and hence $\{[a],[b]\}=\pi(I)$ is independent in $M / \rho_{M}$. This is not possible and we conclude that $M / \rho_{M}$ is simple. The equivalence in (2) follows immediately.

PROPOSITION 4.3. For a matroid $M=(E, \mathcal{I})$, the following three statements are equivalent:

(1) $\rho_{M}=\iota_{M}$ (or equivalently, $M / \rho_{M} \cong M$ ).

(2) $M$ is a simple matroid.

(3) $M$ is a subdirect product of totally disconnected matroids.

Proof. (1) $\Rightarrow(2)$ holds by Proposition (1). For (2) $\Rightarrow(1)$, suppose $M$ is a simple matroid. Let $a, b \in M$ with $a \sim \rho b$. Since $M$ has no 2-circuits, $a=b$ and $\sim \rho$ coincides with $\approx$. Then, for any $a \in I \in \mathcal{I}$, $[a] \cap I=\{a\} \cap I=\{a\}$ which gives $\mathcal{I}=\mathbb{I}_{\rho}$. The equality $\rho_{M}=\iota_{M}$ follows.

(2) $\Longrightarrow$ (3) Suppose $M$ is simple. If $|M| \leq 2$, then $M$ is totally disconnected and we are done. Suppose thus $|M|=n>2$. Choose $x \neq y$ in $M$ and let $M_{x y}$ be the discrete matroid with ground set $\{x, y\}$. Define a function $f: E \rightarrow\{x, y\}$ by

$$
f(t)= \begin{cases}x & \text { if } t=x, \\ y & \text { if } t \in E-x .\end{cases}
$$

Then $f: M \rightarrow M_{x y}$ is a surjective weak map and $M_{x y}$ is a totally disconnected matroid. Let $p_{j}: M \rightarrow M_{j}$ with $j=1,2, \ldots, k$ be the collection of all surjective weak maps from $M$ to a totally disconnected matroid $M_{j}$. Clearly all mappings of the type as $f$ defined above for distinct pairs $x, y$ are included in this collection and $n \leq k$. We show $M$ is a subdirect product of $M_{1}, M_{2}, \ldots, M_{k}$. Let $\alpha_{j}=\operatorname{ker} p_{j}=\left(\sim j, \mathbb{I}_{j}\right)$. Choose $a, b \in M$ with $a \sim_{j} b$ for all $j$. Using a suitable map $f$ as defined above, we can deduce that $a=b$. Let $I=\left\{a_{1}, a_{2}, \ldots, a_{m}\right\} \in \mathcal{I}$. Then $m \leq n \leq k$ and $I=\left\{a_{1}\right\} \cup\left\{a_{2}\right\} \cup \cdots \cup\left\{a_{m}\right\} \in$ $\mathbb{I}_{1} \vee \mathbb{I}_{2} \vee \cdots \vee \mathbb{I}_{m} \subseteq \mathbb{I}_{1} \vee \mathbb{I}_{2} \vee \cdots \vee \mathbb{I}_{k}$ and hence $\mathcal{I} \subseteq \mathbb{I}_{1} \vee \mathbb{I}_{2} \vee \cdots \vee \mathbb{I}_{k}$.

(3) $\Rightarrow$ (2) Suppose $M$ is a subdirect product of the totally disconnected matroids $M_{1}, M_{2}, \ldots, M_{k}$. Let $p_{j}: M \rightarrow M_{j}$ be the associated surjective weak maps with $\operatorname{ker} p_{j}=\alpha_{j}=\left(\sim j, \mathbb{I}_{j}\right)$ for $j=$ $1,2, \ldots, k$. Let $a, b \in M$. If $\{a, b\}$ is a 2-circuit, then $a \neq b$ and hence $p_{j}(a) \neq p_{j}(b)$ for some $j$. Then $\left.p_{j}\right|_{\{a, b\}}:\{a, b\} \rightarrow\left\{p_{j}(a), p_{j}(b)\right\}$ is an injective weak map which gives $\left\{p_{j}(a), p_{j}(b)\right\}$ a circuit in the totally disconnected matroid $M_{j}$. This contradiction means $\{a, b\}$ must be independent and $M$ a simple matroid follows.

As will be done in the next result, which follows immediately from the two previous results, it is sometimes more convenient to write the congruence $\rho_{M}$ as $\rho(M)$. 
COROLLARY 4.4. For any matroid $M, \rho\left(M / \rho_{M}\right)=\iota_{M} / \rho_{M}$.

We will comment on these properties later, but first we give another example.

\subsection{Connected and totally disconnected matroids}

In this subsection we consider another well-known equivalence relation on a matroid $M=(E, \mathcal{I})$. For $x, y \in M, x \sim \gamma y \Leftrightarrow x=y$ or there is a circuit $C$ in $M$ that contains the two distinct elements $x$ and $y$. Let $\mathbb{I}_{\gamma}:=\{I \in \mathcal{I} \mid$ for all $a \in I,[a] \cap I=\{a\}\}$ with $\gamma_{M}:=\left(\sim \gamma, \mathbb{I}_{\gamma}\right)$. Below it will be shown that $\gamma_{M}$ is a congruence on $M$. Clearly $\rho_{M} \sqsubseteq \gamma_{M}$. In the proof of the next result, we will use the Strong Circuit Elimination property (SCE) satisfied by the circuits in a matroid: If $S_{1}$ and $S_{2}$ are circuits with $x \in S_{1}-S_{2}$ and $y \in S_{1} \cap S_{2}$, then there is a circuit $S_{3} \subseteq S_{1} \cup S_{2}$ with $x \in S_{3}$ and $y \notin S_{3}$.

PROPOSITION 4.5. For a matroid $M=(E, \mathcal{I}), \gamma_{M}:=\left(\sim_{\gamma}, \mathbb{I}_{\gamma}\right)$ is a strong congruence on $M$.

Proof. (C1) It is a well-established result that $\sim \gamma$ is an equivalence relation, but we reproduce the proof of the transitivity here for completeness. Let $x, y$ and $z$ be distinct elements of $M$ with $x \sim_{\gamma} y$ and $y \sim \gamma z$. Let $C_{1}$ and $C_{2}$ be circuits with $x, y \in C_{1}$ and $y, z \in C_{2}$. If $z \in C_{1}$ or $x \in C_{2}$ we are done, so suppose $x \in C_{1}-C_{2}$ and $z \in C_{2}-C_{1}$. Let $\left|C_{2}-C_{1}\right|=n$ and we proceed by induction on $n$ where the induction hypothesis is:

If $S_{1}$ and $S_{2}$ are circuits in $M$ with $a \in S_{1}-S_{2}, b \in S_{2}-S_{1}, S_{1} \cap S_{2} \neq \varnothing$ and $\left|S_{2}-S_{1}\right|=n$, then there is a circuit $S_{3}$ in $M$ with $a, b \in S_{3}$.

If $\left|C_{2}-C_{1}\right|=1$, then $C_{2}-C_{1}=\{z\}$. By the (SCE), there is a circuit $C_{3} \subseteq C_{1} \cup C_{2}$ with $x \in C_{3}$ and $y \notin C_{3}$. We can't have $C_{3}$ contained in $C_{1}$ for such a containment must then be proper since $y \in C_{1}-C_{3}$. Thus $z \in C_{3}$ and we are done. Let $n>1$, suppose the statement is true for all $1 \leq k<n$ and let $\left|C_{2}-C_{1}\right|=n$. Again, by the $(S C E)$, there is a circuit $C_{4} \subseteq C_{1} \cup C_{2}$ with $x \in C_{4}$ and $y \notin C_{4}$. If $z \in C_{4}$ we are done, so suppose $z \notin C_{4}$. Since $y \in C_{1}-C_{4}$, we can't have $C_{4} \subseteq C_{1}$. Choose $t \in C_{2}-C_{1}$ with $t \in C_{4}$. Apply the (SCE) to $z \in C_{2}-C_{4}$ and $t \in C_{2} \cap C_{4}$ to get a circuit $C_{5} \subseteq C_{2} \cup C_{4}$ with $z \in C_{5}$ and $t \notin C_{5}$. If $x \in C_{5}$ we are done, so suppose $x \notin C_{5}$. Now $C_{1} \cap C_{5} \neq \varnothing$ (if $C_{1} \cap C_{5}=\varnothing$, then we get the contradiction that $C_{5}$ is a proper subset of $C_{2}$ since $t \in C_{2}-C_{5}$ ). We can thus apply the induction assumption to $x \in C_{1}-C_{5}, z \in C_{5}-C_{1}, C_{1} \cap C_{5} \neq \varnothing$ and $\left|C_{5}-C_{1}\right|<\left|C_{2}-C_{1}\right|=n$ to get a circuit $C_{6}$ with $x, z \in C_{6}$.

(C2) By definition, $\mathbb{I}_{\gamma} \subseteq \mathcal{I}$ and the conditions (M1) and (M2) for showing $M_{\gamma}=\left(E, \mathbb{I}_{\gamma}\right)$ is a matroid follow immediately. Again we first proof the Substitution Property which is required for the justification of the Independence augmentation axiom.

(C3) Let $A=\left\{a_{1}, a_{2}, \ldots, a_{n}\right\} \in \mathbb{I}_{\gamma}, B=\left\{b_{1}, b_{2}, \ldots, b_{n}\right\} \subseteq M$ with $|A|=|B|=n$ and $a_{i} \sim_{\gamma} b_{i}$ for all $i=1,2, \ldots, n$. Note that since $A \in \mathbb{I}_{\gamma}$, we know that $a_{i}$ is not related to $a_{j}$ for any $i \neq j$. This, in turn, means that $b_{i}$ is not related to $b_{j}$ for $i \neq j$ (since $a_{i} \sim_{\gamma} b_{i}$ for all $i$ ). Thus $\left[b_{i}\right] \cap B=\left\{b_{i}\right\}$ for all $i$. For $B \in \mathbb{I}_{\gamma}$ we still need $B \in \mathcal{I}$. If $B$ is dependent, then it contains a circuit $C$ with at least two elements, say $b_{i} \neq b_{j}$. But this gives the contradiction $b_{i} \sim_{\gamma} b_{j}$. Thus $B \in \mathcal{I}$ and $B \in \mathbb{I}_{\gamma}$ follows.

(M3) To complete the proof that $M_{\gamma}=\left(E, \mathbb{I}_{\gamma}\right)$ is a matroid, an argument similar to the corresponding part in the proof of Proposition 4.1 will do the job.

(C4) This requirement is given by the definition of $\mathbb{I}_{\gamma}$.

(C5) Let $U$ be an independent subset of $M, B$ a basis for $U$ in the matroid $M_{\gamma}=\left(E, \mathbb{I}_{\gamma}\right)$ and $x \in M$ with $[x] \cap U=\varnothing$. Suppose $B \cup x \in \mathbb{I}_{\gamma}$. We need to show that $U \cup x \in \mathcal{I}$. If $U \cup x$ is dependent, then it contains a circuit $C$ which necessarily must contain $x$. Moreover, if $c \in C-\{x\}$, then $c \in[x] \cap U=\varnothing$ which gives the contradiction $C=\{x\}$. Thus $U \cup x \in \mathcal{I}$.

A matroid $M$ is connected if any two distinct elements of $M$ are contained in a common circuit, i.e., $x \sim \gamma y$ for all $x, y \in M$. If this is not the case, $M$ is called disconnected. An equivalence class $[x]$ in a matroid with respect to the equivalence relation $\sim \gamma$ is called a connected component of $M$. It can easily be seen that $M$ is totally disconnected if and only if it has no nontrivial connected components. Any totally disconnected matroid is disconnected. 
PROPOSITION 4.6. Let $M=(E, \mathcal{I})$ be a matroid with $\gamma_{M}:=\left(\sim \gamma, \mathbb{I}_{\gamma}\right)$ the congruence on $M$ as defined above. Then:

(1) $M$ is connected $\Leftrightarrow \gamma_{M}=v_{M}$ (or equivalently, $M / \gamma_{M}$ is trivial).

(2) $M$ is totally disconnected $\Leftrightarrow \gamma_{M}=\iota_{M}$ (or equivalently, $M / \gamma_{M} \cong M$ ).

(3) $M / \gamma_{M}$ is totally disconnected; hence $\gamma\left(M / \gamma_{M}\right)=\iota_{M} / \gamma_{M}$.

Proof. (1) and (2) are straightforward; we check (3). Let $\pi_{\gamma}: M \rightarrow M / \gamma_{M}$ be the canonical quotient map. If $M / \gamma_{M}$ has a circuit $C_{1}$, choose $J \subseteq M$ such that $\left.\pi_{\gamma}\right|_{J}: J \rightarrow \pi_{\gamma}(J)=C_{1}$ is injective. We know $\gamma=\operatorname{ker} \pi_{\gamma}$ and hence $J \notin \mathbb{I}_{\gamma}$. Actually, $J \notin \mathcal{I}$, for $J \in \mathcal{I}-\mathbb{I}_{\gamma}$ means there is an element $b \in[a] \cap J$ for some $a \in J, a \neq b$. But $b \sim_{\gamma} a$ means $\pi_{\gamma}(a)=\pi_{\gamma}(b)$ which contradicts $\left.\pi_{\gamma}\right|_{J}$ injective. Thus $J$ is dependent and it contains a circuit, say $C$. Then $\left.\pi_{\gamma}\right|_{C}: C \rightarrow \pi_{\gamma}(C)$ is injective which gives a circuit $C_{2}$ with $C_{2} \subseteq \pi_{\gamma}(C) \subseteq \pi_{\gamma}(J)=C_{1}$. But this is only possible if $C_{2}=\pi_{\gamma}(C)=\pi_{\gamma}(J)=C_{1}$ and so $\left.\pi_{\gamma}\right|_{C}: C \rightarrow \pi_{\gamma}(C)=C_{1}$. Since $C$ is a circuit in $M$, all the elements of $C$ are related and thus $C_{1}=\pi_{\gamma}(C)=\{[c]\}$ for a $c \in C$; a contradiction. Hence $M / \gamma_{M}$ contains no circuits, i.e., it is totally disconnected.

In line with the properties of the previous example, we should mention the known result that a totally disconnected matroid is disconnected; hence it can be decomposed as a direct sum of matroids (in fact, a matroid is disconnected if and only if it can be decomposed as a direct sum).

In conclusion, we motivate our earlier remark why these two examples suggest that it may be possible to accommodate them in a general theory of connectednesses and disconnectednesses for matroids using congruences along the lines of what was done for topological spaces [9] and graphs [8] and motivated by their algebraic counterparts. For such a theory, there is a function $\eta$ that assigns to each matroid $M$ a congruence $\eta_{M}$. Let $C:=\left\{\right.$ matroids $M \mid \eta_{M}=v_{M}$ \} and $D:=\left\{\right.$ matroids $\left.M \mid \eta_{M}=\iota_{M}\right\}$. Typically then, $\eta_{M}$ gives a partition of $M$ for which the submatroids determined by the congruence classes are in $C, M / \eta_{M}$ is in $D$ and both $\eta_{M}$ and $M / \eta_{M}$ are maximal in some sense. Moreover, there is a Galois correspondence between the classes $C$ and $D$, and the elements of $D$ usually has a decomposition as a subdirect product of certain distinguished matroids. Elements of such a theory are clearly present for the two examples above as shown by their properties.

\section{REFERENCES}

[1] Arhangel'skiı̆, A. V. And Wiegandt, R. Connectednesses and disconectednesses in topology. General Topology and Appl. 5 (1975), 9-33.

[2] Broere, I., Heidema J. and Veldsman, S. Congruences and Hoehnke radicals on graphs. Discuss. Math. Graph Theory 40 (4) (2020), 1067-1084.

[3] Fried, E. and Wiegandt, R. Connectednesses and disconnectednesses of graphs. Algebra Univ. 5 (1975), 411-428.

[4] Gardner, B. J. And Wiegandt, R. The Radical Theory of Rings. Marcel Dekker Inc., 2004.

[5] Gordon, G. And McNulty, J. Matroids: a geometric introduction. University Press, Cambridge, 2012.

[6] OxLey, J. G. Matroid Theory. Oxford University Press, New York, 1992.

[7] Veldsman, S. Congruences on topological spaces with an application to radical theory. Alg. Universalis 80 (2019), article 25.

[8] Veldsman, S. Connectednesses of graphs and congruences. Asian-European fournal of Mathematics 14(10), (2021).

[9] Veldsman, S. Topological connectednesses and congruences. Quaestiones Mathematicae. https://doi.org/10.2989/16073606.2020.1825020 (2020).

Open Access statement. This is an open-access article distributed under the terms of the Creative Commons AttributionNonCommercial 4.0 International License (https://creativecommons.org/licenses/by-nc/4.0/), which permits unrestricted use, distribution, and reproduction in any medium for non-commercial purposes, provided the original author and source are credited, a link to the CC License is provided, and changes - if any - are indicated. 